\begin{tabular}{|l|c|c|c|c|}
\hline $\begin{array}{l}\text { Cuadernos de Investigación Geográfica } \\
\text { Geographical Research Letters }\end{array}$ & 2019 & No $^{\circ} 45(2)$ & pp. 571-600 & eISSN 1697-9540 \\
\hline
\end{tabular}

\title{
METODOLOGÍA DE EVALUACIÓN DEL RIESGO DE INCENDIOS FORESTALES Y PRIORIZACIÓN DE TRATAMIENTOS MULTIFUNCIONALES EN PAISAJES MEDITERRÁNEOS
}

\author{
F.J. ALCASENA-URDÍROZ1*, C. VEGA-GARCÍA ${ }^{1,2}$, A.A. AGER ${ }^{3}$, \\ M. SALIS ${ }^{4,5}$, N.J. NAUSLAR ${ }^{6,7}$, F.J. MENDIZABAL ${ }^{8}$, R. CASTELL ${ }^{9}$ \\ ${ }^{1}$ Departament d'Enginyeria Agroforestal, Escola Tècnica Superior d'Enginyeria Agrària, \\ Universitat de Lleida, Alcalde Rovira Roure 191, 25198 Lleida, España. \\ ${ }^{2}$ Centre Tecnològic Forestal de Catalunya, Carretera de Sant Llorenç \\ de Morunys km 2, 25280 Solsona, España. \\ ${ }^{3}$ USDA Forest Service, Rocky Mountain Research Station, \\ 72510 Coyote Road Pendleton, 97801 Oregon, USA. \\ ${ }^{4}$ Euro-Mediterranean Center on Climate Change, IAFES Division, \\ Via Enrico De Nicola 9, I-07100 Sassari, Italia. \\ ${ }^{5}$ National Research Council, Institute of Biometeorology, Regione Baldinca, 07100 Sassari, Italia. \\ ${ }^{6}$ Cooperative Institute for Mesoscale Meteorological Studies, University of Oklahoma, \\ Norman, 73019 Oklahoma, USA. \\ ${ }^{7}$ NOAA/NWS/NCEP Storm Prediction Center, Norman, 73019 Oklahoma, USA. \\ ${ }^{8}$ Servicio de Riqueza Territorial y Tributos Patrimoniales, Gobierno de Navarra, \\ Carlos III n ${ }^{\circ}$ 4, 31002 Pamplona, España. \\ ${ }^{9}$ Bomberos de Navarra, Agencia Navarra de Emergencias, \\ Calle Aoiz 35 bis $3^{\circ}, 31004$ Pamplona, España.
}

RESUMEN. En las regiones mediterráneas, el efecto combinado del éxodo rural, la falta de gestión forestal y las politicas de supresión de incendios han contribuido notablemente en el aumento de la carga y continuidad de los combustibles forestales sobre extensas áreas. El resultado es una creciente incidencia de incendios forestales que supera la capacidad de extinción. Debido a una limitada disponibilidad de recursos económicos para la gestión del paisaje, resulta necesario priorizar la protección de los bienes con una expectativa de pérdida elevada y el tratamiento del combustible en puntos estratégicos para contener los incendios que impactan en núcleos urbanos. Este estudio se desarrolla en el Valle de Juslapeña (Navarra, España) para demostrar la priorización de actuaciones en la gestión de combustibles. En el área de estudio, los frecuentes y grandes incendios forestales han causado notables daños en el patrimonio forestal y los bienes de las comunidades rurales. Primero, se generó la cartografía de riesgo de incendios para los bienes de elevado valor, a continuación, se diseñó el mosaico óptimo de tratamientos dentro de la cuenca de exposición en base a la exposición de las masas arboladas y la transmisión a viviendas residenciales. A su vez, se identificaron los rodales capitalizados en existencias donde las extracciones podrían 
abastecer las necesidades de la población local o costear parcialmente el coste de los tratamientos. Según se observa, las mayores pérdidas se obtuvieron en las viviendas localizadas al sur del área de estudio debido a su elevada probabilidad de quema. Los incendios iniciados fuera del área de estudio también afectaron a las viviendas residenciales y, por tanto, la extensión de los planes de gestión de incendios debe ser ajustada considerando el origen y la escala del riesgo en los núcleos urbanos. La metodología que se presenta en este estudio puede ser adaptada a la gestión multifuncional de cualquier otra región mediterránea con un elevado riesgo de incendios.

\section{Forest fire risk assessment and multifunctional fuel treatment prioritization methods in Mediterranean landscapes}

ABSTRACT. In Mediterranean areas, the combined effects of the rural exodus, lack of forest management, and fire suppression policies have substantially contributed to increased forest fuel loadings and continuity over large areas. The result is a growing incidence of wildfires that exceed fire suppression capacity. Economic resources for landscape management are limited, and thus they must be prioritized towards the protection of valued assets where there is a high expectation of loss and the fuel treatments on strategic locations that restrict fires spreading into communities. We completed a case study in the Juslapeña Valley (Navarra, Spain) to demonstrate prioritization of fuel management activities. The study area has frequent and large forest fires that have caused significant damage to forest values and assets in rural communities. We first generated a wildfire risk map for valued assets, and then designed the optimal treatment mosaic within the community fireshed considering the wildfire exposure to forestlands and fire transmission to residential housing. We also identified overstocked stands where the timber or firewood production might supply the needs of local communities and partially cover the treatment cost. We found that the highest economic losses were obtained in residential houses located in the southern portion of the study area, mainly due to a higher burn probability. Fires ignited outside of the study area also exposed communities, and thus the extent considered in wildfire management plans needs to be adjusted to reflect the source and scale of risk to communities. The assessment framework presented in this study can be adapted to the multi-functional forest management in any fire-prone Mediterranean region elsewhere.

Palabras clave: evaluación de riesgo, optimización espacial, tratamiento de combustibles, gestión multifuncional, cuencas de exposición.

Key words: risk assessment, spatial optimization, fuels treatment, multi-functional management, community fireshed.

Recibido: 22 de Agosto de 2018

Aceptado: 2 de Enero de 2019 
*Correspondencia: Fermín J. Alcasena Urdíroz, Departament d'Enginyeria Agroforestal, Universitat de Lleida, Alcalde Rovira Roure 191, 25198 Lleida, España. E-mail: ferminalcasena@eagrof.udl.cat

\section{Introducción}

El creciente incremento en la carga y continuidad de combustibles forestales en los ecosistemas europeos de clima templado y mediterráneo durante las últimas décadas ha facilitado la ocurrencia de grandes incendios forestales, constatando la rápida evolución del régimen de incendios a un nuevo régimen post-industrial sometido a las condiciones meteorológicas extremas (Pausas y Fernández-Muñoz, 2012; Seijo y Gray, 2012). Factores como el éxodo rural, la falta de gestión forestal y una política de supresión total de incendios son los principales factores que explican la creciente acumulación de biomasa en el territorio y la consecuente desaparición del paisaje cultural en mosaico donde la discontinuidad de combustibles limitaba la propagación del fuego (Cervera et al., 2016; Poyatos et al., 2003). En los países europeos meridionales en torno a 48.000 incendios forestales queman anualmente unas 448.000 hectáreas de media (1980 a 2015), situando a España como el país más afectado en esta región con el $45 \%$ del área quemada (San-Miguel-Ayanz et al., 2017). A su vez, son los escasos $(<10 \%)$ pero grandes incendios forestales ( $>100 \mathrm{ha})$ son los responsables de la mayor parte del área quemada $(>60 \%)$ en el periodo estival y están asociados a condiciones meteorológicas de sequía acumulada, fuertes vientos y bajas humedades relativas (San-Miguel-Ayanz et al., 2013). Estos incendios forestales recorren largas distancias $(>10 \mathrm{~km})$, presentan comportamientos extremos que superan la capacidad de extinción (fuegos activos de copas con múltiples saltos de fuego), están asociados a episodios de simultaneidad, y frecuentemente impactan núcleos urbanos habitados localizados en la interfaz urbano forestal (Castellnou y Miralles, 2009). Además, la mayor parte del área quemada resulta severamente afectada y se requiere de costosos trabajos de restauración y monitoreo post-incendio encaminados a minimizar los efectos negativos en los bienes socioeconómicos y de interés natural (Moya et al., 2014; Prats et al., 2014). En cuanto a la causalidad se refiere, el origen de las igniciones es mayoritariamente antrópico y los incendios de rayo se concentran en cadenas montañosas donde difícilmente superan el 20\% del total (Costafreda-Aumedes et al., 2016; Rodrigues y De la Riva, 2014).

El riesgo de incendios se define como la expectativa de pérdida o beneficio en un bien o servicio que es afectado por el fuego, es espacialmente explícito y se puede evaluar cuantitativamente (Ager et al., 2010; Finney, 2005; Scott et al., 2013). Está integrado por la probabilidad de quema y sus consecuencias, siendo las consecuencias el resultado de la susceptibilidad del bien o servicio afectado por el fuego a una determinada intensidad. La exposición no contempla los efectos del fuego y se obtiene a partir de la intensidad y probabilidad de quema (Miller y Ager, 2013). Los efectos del fuego se pueden aproximar con funciones de susceptibilidad o modelos de mortalidad en el caso de especies arboladas (Fernandes et al., 2012; Thompson et al., 2011). La transmisión consiste en asignar a las coordenadas o polígono de origen del fuego los 
bienes o servicios expuestos (por ej., número de viviendas) dentro de su perímetro y nos permite delimitar el contorno y extensión de las cuencas de exposición ("firesheds"). La cuenca de exposición por tanto es el área de terreno donde las igniciones ocurridas en condiciones meteorológicas típicas asociadas al periodo de incendios originan fuegos que, en propagación libre, alcanzan un determinado bien de interés natural o elevado valor económico (por ej., núcleo urbano o hábitat de protección prioritaria) (Ager et al., 2016a; Thompson et al., 2013). Por otra parte, la zona de ignición en viviendas ("home ignition zone") es el entorno próximo a las estructuras (buffer de 30-60 m) donde la intensidad del fuego determina en mayor medida los daños y su pérdida o destrucción (Cohen, 2008). Los puntos estratégicos de gestión (PEG) en el paisaje son aquellas parcelas o rodales a tratar preferentemente donde la reducción de combustibles facilita la labor de los medios de extinción (aplicación del fuego táctico) y contiene significativamente el potencial del gran incendio forestal (Ager et al., 2011; Costa et al., 2011). Su localización y extensión se puede determinar en base al criterio experto, a partir del estudio de incendios históricos (González-Olabarria et al., 2019), o alternativamente mediante el empleo de simuladores y modelos de optimización espacial (Ager et al., 2016b; Finney, 2007).

Los simuladores nos permiten predecir el comportamiento y la propagación de los grandes incendios forestales (Arca et al., 2007; Jahdi et al., 2016; Salis et al., 2016a). A su vez, algoritmos altamente eficientes (Finney, 2002) nos permiten saturar el paisaje con miles de igniciones ( $>10^{4}$ igniciones) y determinar en alta resolución $(<50 \mathrm{~m}$ de píxel) la intensidad del fuego y su probabilidad de quema (Alcasena et al., 2015). Actualmente, el acceso a los datos requeridos para la simulación de incendios es cada vez mayor y los avances tecnológicos permiten caracterizar con precisión las variables geoespaciales del paisaje (topografía, modelos de combustible de superficie y parámetros de copas) en alta resolución (González-Olabarria et al., 2012; Marino et al., 2016). A partir de los registros en estaciones meteorológicas resulta posible determinar localmente cuáles son las condiciones meteorológicas extremas asociadas a los grandes incendios forestales y caracterizar así los escenarios más frecuentes durante el periodo estival (Bradshaw y McCormick, 2000; Duane y Brotons, 2018). Además, a partir de las igniciones históricas se pueden generar modelos de ocurrencia que nos permitan replicar el patrón de igniciones requerido como dato de entrada en las simulaciones (Alcasena et al., 2017; Alcasena et al., 2016a).

La mayoría de los estudios previos desarrollados en ambientes mediterráneos se limitan a cuantificar el riesgo y la exposición de incendios (Alcasena et al., 2016b; Palaiologou et al., 2018; Salis et al., 2013), y muy pocos evalúan las diferencias entre posibles estrategias (configuraciones espaciales e intensidades de tratamientos) encaminadas a mitigar las pérdidas por incendios (Oliveira et al., 2016; Salis et al., 2016b). Debido a la escasez de recursos económicos disponibles para la ejecución de trabajos de prevención y la existencia de objetivos contrapuestos, la optimización espacial integra un complejo análisis que nos permite identificar una solución de compromiso con múltiples objetivos en las parcelas a tratar (Vogler et al., 2015). Esto es posible ya que el tratamiento de combustibles (es decir, claras, quemas y desbroces) se pueden compatibilizar con labores de restauración de hábitats de especial interés, mejora de pastos y aprovechamientos 
forestales (Lasanta et al., 2018). A tal efecto, resulta imprescindible proporcionar los resultados a escala de rodal (es decir, identificación de parcelas a tratar) para facilitar la transferencia de los resultados a los gestores del territorio responsables de la ejecución de los tratamientos (Alcasena et al., 2018). En ocasiones, los trabajos de restauración implican importantes cortas de madera cuando se ejecutan en extensas masas arboladas y las extracciones de madera para la apertura de discontinuidades pueden aportar beneficios económicos que permitan cubrir al menos parcialmente el coste de los tratamientos (Ager et al., 2016b).

Para mitigar el riesgo de incendios es necesario generar paisajes resistentes al fuego, aplicar medidas encaminadas a prevenir igniciones antrópicas, hacer un uso eficiente de los medios de extinción, reducir la susceptibilidad al fuego en los bienes de elevado valor y fomentar una percepción responsable del riesgo en la población local, especialmente en áreas de interfaz urbano forestal (Alcasena et al., 2019; Paveglio et al., 2016). Concretamente, es la gestión de combustibles la medida principal en lo que a la creación de núcleos urbanos y paisajes resistentes al fuego se refiere. A su vez, el tratamiento de combustibles es especialmente útil en entornos próximos a los bienes de interés y para reducir localmente la intensidad del fuego, o en puntos estratégicos del territorio para limitar la propagación de grandes incendios, reduciendo así la probabilidad de quema a gran escala de paisaje. El objetivo del presente estudio es (1) evaluar el riesgo de incendios (es decir, cuantificar la expectativa de pérdidas económicas en caso de gran incendio forestal) usando cartografía de alta resolución para los principales bienes (viviendas y repoblaciones de carácter productor) del Valle de Juslapeña (Navarra, España), e (2) identificar en el paisaje los rodales preferentes a tratar para mitigar la transmisión de incendios a núcleos urbanos, reducir las pérdidas potenciales en masas forestales y maximizar las extracciones en los tratamientos (leñas o madera), conformando con ello una metodología efectiva para la definición en el territorio estudiado de tratamientos multifuncionales y puntos estratégicos de gestión. Finalmente, en base a los resultados obtenidos, se proponen una serie de medidas que permitan la reducir el riesgo de incendios en el área de estudio.

\section{Material y métodos}

\subsection{Esquema general}

El esquema general empleado en este estudio integra dos procesos diferenciados, uno para evaluar el riesgo de incendios y otro encaminado a priorizar espacialmente el tratamiento de combustibles (Fig. 1). Ambos requieren resultados cuantitativos procedentes de la simulación de incendios forestales a escala de paisaje. El riesgo de incendios se determinó como la expectativa de pérdida económica en bienes de elevado valor (viviendas residenciales y pinares productores). En base a los objetivos fijados en la gestión forestal (y la priorización establecida entre objetivos) se diseñaron diferentes mosaicos de tratamientos multifuncionales en el área de planificación (cuenca de exposición) con un modelo de optimización, para finalmente analizar su correspondencia espacial. El trazo de línea discontinuo presenta un bucle de retorno 
que permitiría evaluar el efecto de los tratamientos estratégicos preventivos (es decir, soluciones multifuncionales) en la disminución de pérdidas económicas. La metodología empleada en este estudio puede adaptarse a las necesidades locales (objetivos de la gestión forestal) y aplicarse en cualquier otro lugar donde los incendios suponen un riesgo para los bienes y servicios.

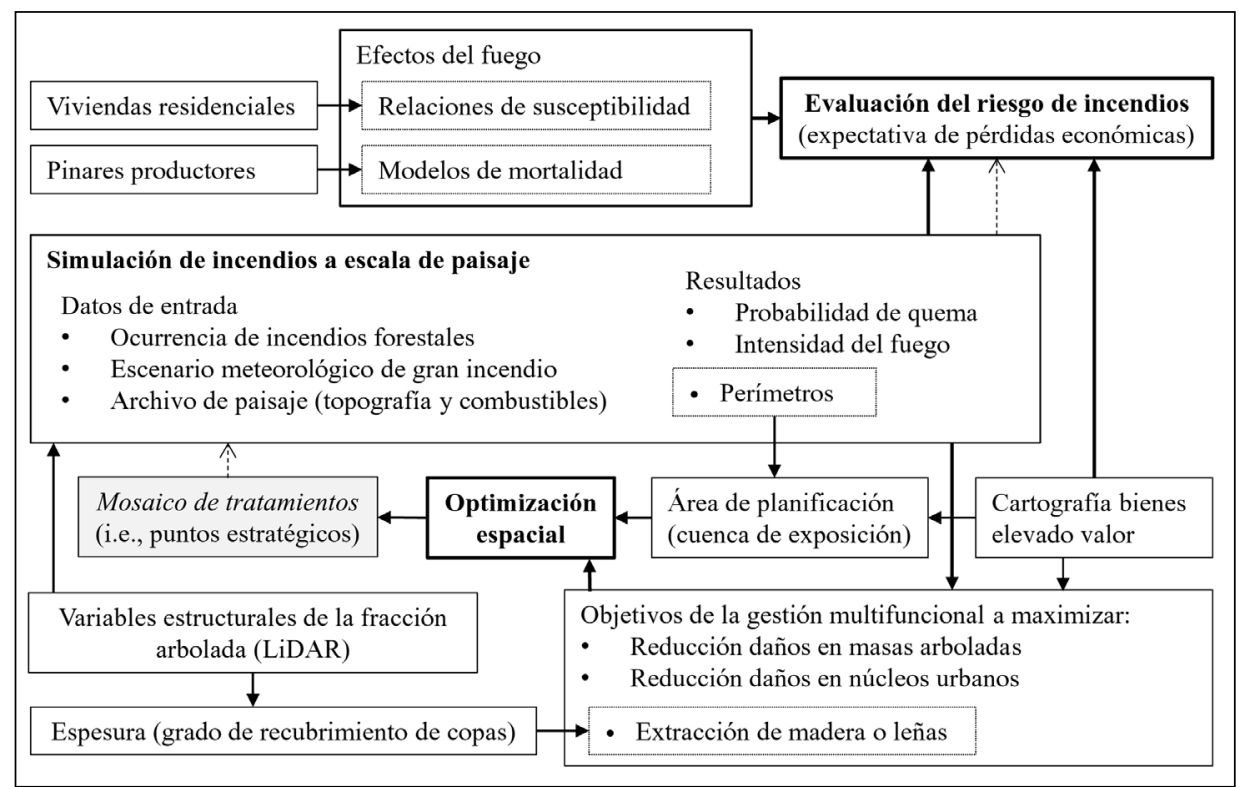

Figura 1. Esquema general de evaluación de riesgo y optimización espacial de tratamientos aplicado en el estudio. Los resultados de la simulación de incendios se emplean para cuantificar el riesgo de incendios, determinar el área de planificación y facilitar la priorización de tratamientos (es decir, métricas cuantitativas asociadas a los objetivos de gestión).

\section{2. Área de estudio}

El área de estudio se corresponde con el término municipal del Valle de Juslapeña (Navarra, España), tiene una extensión de 3163 ha y se encuentra situado en el límite norte de la Cuenca de Pamplona (Fig. 2A). La población es de 548 habitantes y se encuentra distribuida en 14 núcleos urbanos. El clima es mediterráneo transicional, con precipitaciones medias anuales en torno a los $1000 \mathrm{~mm}$ y un periodo de sequía estival de unos 3 meses, con temperaturas máximas diarias por encima de los $30^{\circ} \mathrm{C}$. Los campos de cultivo de cereal en secano ocupan los suelos arcillosos y profundos de los fondos de valle. Los pastos mesoxerófitos (Bromus erectus Huds. y Brachypodium pinnatum L.) y las orlas de matorral (Juniperus communis L., Prunus spinosa L., Buxus sempervirens L. y Genista scorpius L.) se sitúan entre los campos de cultivo y zonas arboladas, llegando a ocupar importantes extensiones en el caso de algunos pastos 
comunales. Aunque el roble pubescente (Quercus pubescens Willd.) es la especie arbórea dominante en los bosques procedentes de regeneración natural, también existen múltiples repoblaciones de pino laricio de austria (Pinus nigra Arn. ssp. nigra). En el límite norte, son los hayedos (Fagus sylvatica L.) los que ocupan las exposiciones norte y cotas más elevadas. Los cambios en los usos del suelo durante los últimos 50 años evidencian el incremento de la superficie forestal arbolada, un incremento en la cobertura de matorrales y la reducción de pastizales o zonas abiertas (Fig. 2B y 2C). En los robledales se puede apreciar un claro incremento de la fracción de cabida cubierta así como su expansión en pastizales limítrofes. Las parcelas de cultivo se concentran ahora en los fondos de valle y gran parte de las antiguas roturas difícilmente mecanizables fueron repobladas con pino laricio. Únicamente los pastos comunales habilitados para su aprovechamiento en extensivo (es decir, que disponen de cierres ganaderos y abrevaderos) mantienen las zonas abiertas, en el resto de los casos el incremento de la cobertura de matorral y regenerado natural es muy notable. Estas nuevas masas en edad de monte bravo y latizal bajo son muy vulnerables a incendios debido a la elevada carga y continuidad de combustibles. En el área de estudio los incendios se concentran en verano y las causas principales son la quema de pastos y matorral, así como la quema de rastrojos de cereal (Fig. 3).

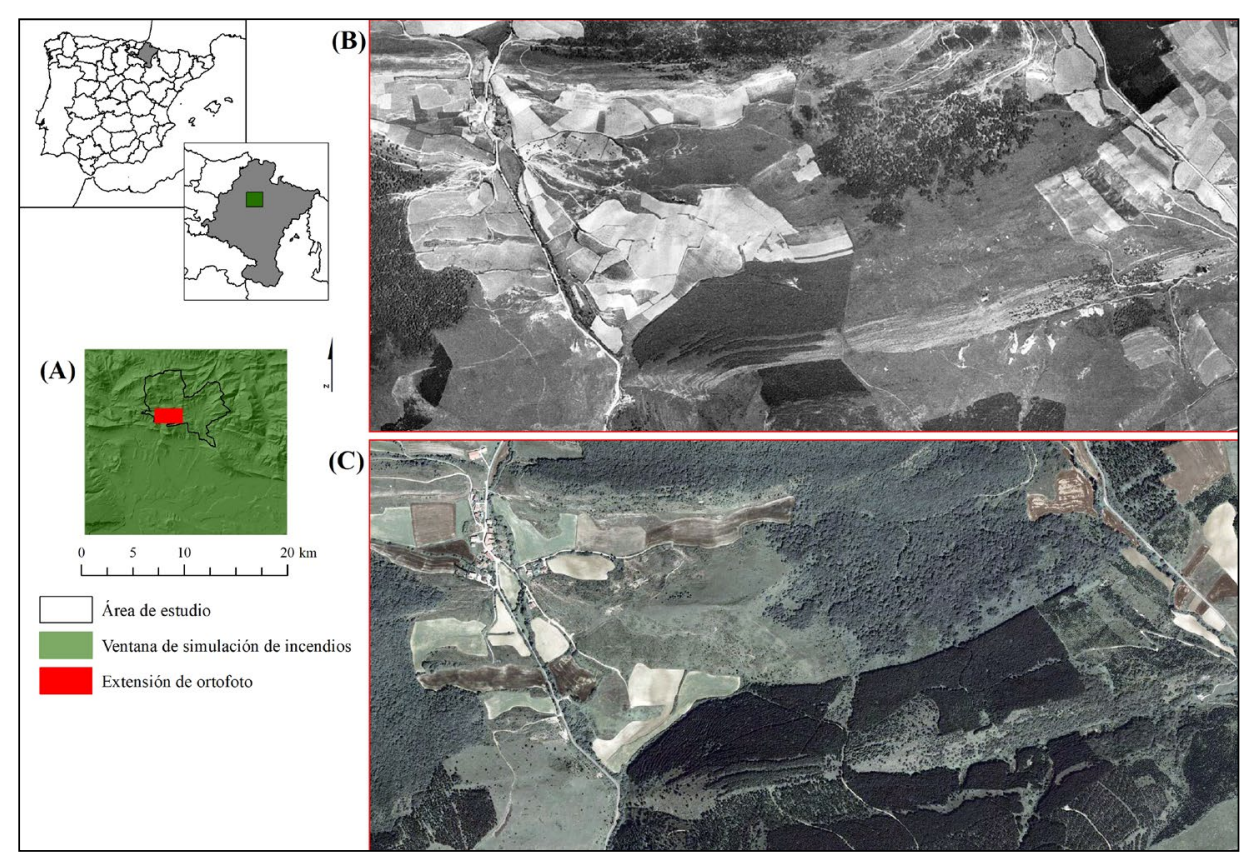

Figura 2. Cambios en el uso del suelo en el área de estudio (A). Las diferencias entre las ortofotos de 1956 (B) y 2016 (C) evidencian el rápido incremento en la carga y continuidad de los combustibles durante los últimos 50 años. 


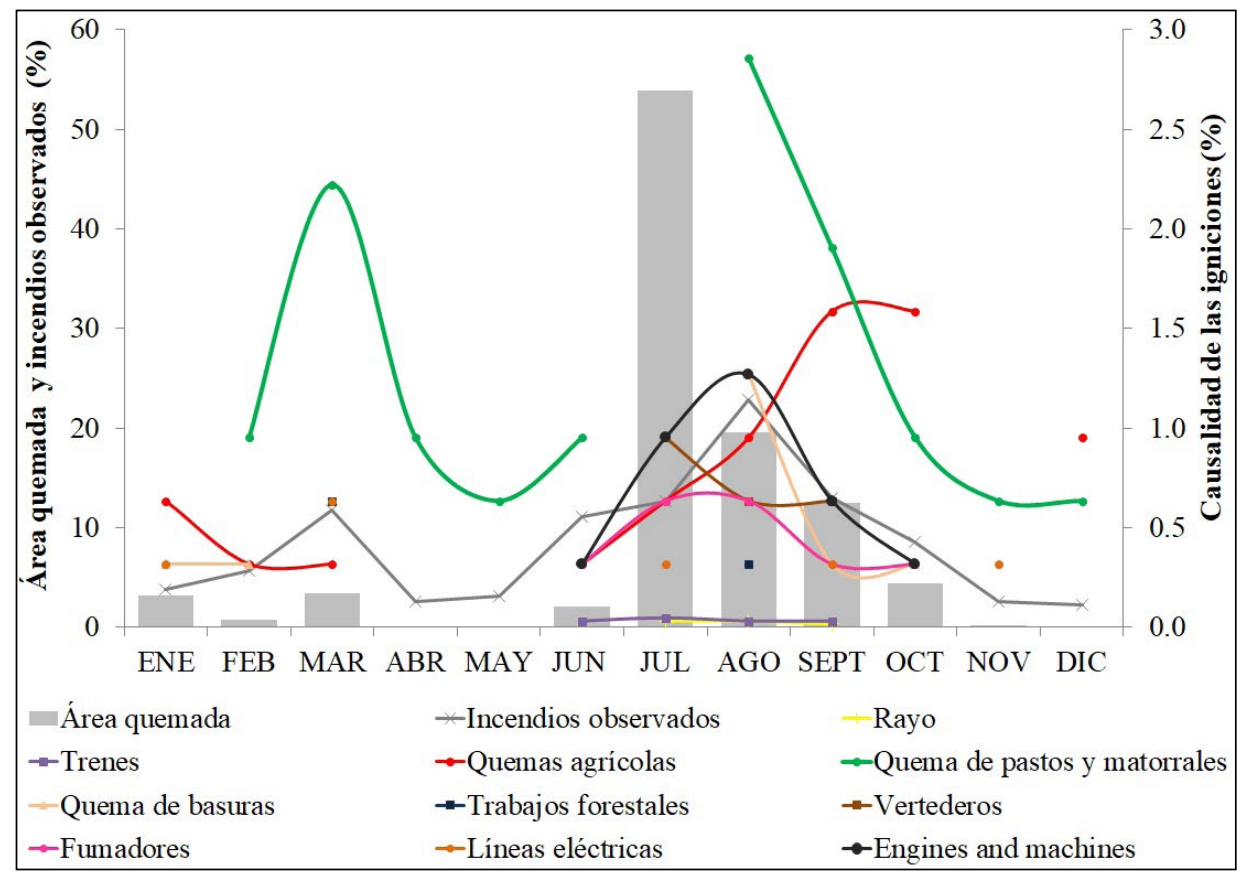

Figura 3. Estacionalidad y causalidad de incendios en el área de estudio. La mayor parte del área quemada se concentra durante el periodo estival y las quemas (pastos y agrícolas) son la causa más frecuente (1985 a 2013).

\subsection{Ocurrencia de incendios}

Los modelos de ocurrencia permiten predecir dónde es más probable que se inicie un incendio y generar un patrón de igniciones que posteriormente pueda ser empleado como dato de entrada en la simulación de incendios (listado de igniciones con sus coordenadas). Existen diferentes métodos para la generación de estos modelos, siendo los modelos de aprendizaje automático, la regresión logística y las redes neuronales los más empleados (Martínez et al., 2009; Rodrigues y De la Riva, 2014; Vega-García et al., 1995). Para ello, se emplean los incendios históricos (coordenadas) y una muestra de puntos (coordenadas) de no-igniciones, a partir de los cuáles se extraen del territorio una serie de variables de carácter geoespacial con las que se construye el modelo. Habitualmente, las variables asociadas a la actividad humana y sus transformaciones del paisaje son las que más contribuyen a explicar estos modelos, puesto que las actividades antrópicas son causantes de la mayor parte de las igniciones conocidas en ambientes Mediterráneos (Costafreda-Aumedes et al., 2018). Para este estudio se consideró un área de ocurrencia de 36.000 ha abarcando el Valle de Juslapeña y los municipios más próximos de su entorno. Se contabilizaron un total de 200 igniciones (1985 a 2013) (MAAyMA, 2015), que además de otras 200 no-igniciones localizadas aleatoriamente, fueron empleadas para extraer una muestra de las variables geoespaciales correspondientes a usos 
de suelo, distancia a carreteras, distancia a núcleos urbanos, densidad de población y distancia a líneas eléctricas. Con el empleo de una red neuronal de correlación en cascada (Fahlman y Lebiere, 1990) se generó un modelo de ocurrencia (Alcasena et al., 2017). Al aplicar el modelo en cada uno de los píxeles del área de ocurrencia, se generó un mapa de probabilidad de ignición (valores entre 0 y 1) a 30 m de resolución (Fig. 4).

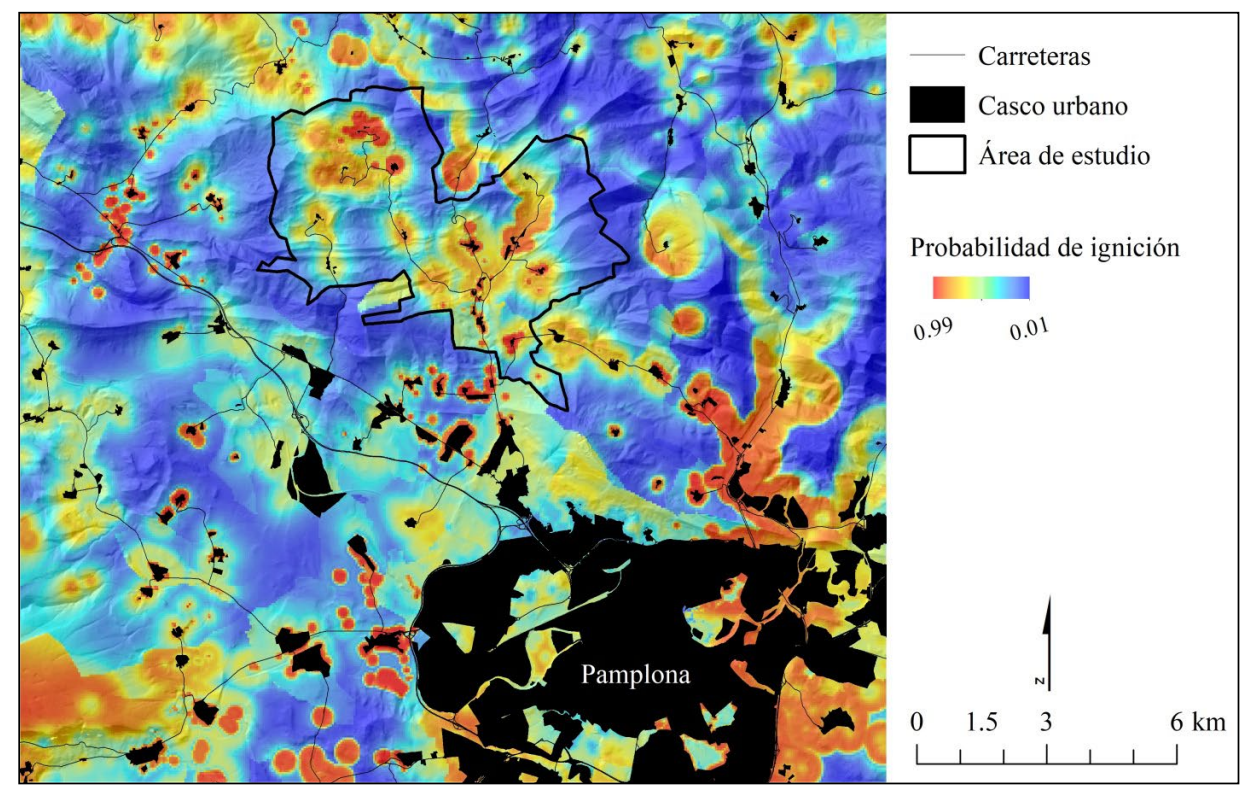

Figura 4. Mapa de probabilidad de ignición en alta resolución $(30 \mathrm{~m})$ generado con redes neuronales a partir de la localización de las igniciones históricas (Alcasena et al., 2017). El mapa fue empleado para generar un patrón con 10.000 puntos de ignición, dato de entrada para la simulación de incendios.

\subsection{Simulación de incendios}

Para la simulación de los incendios se requiere la información geoespacial que caracteriza el paisaje, las condiciones meteorológicas típicas asociadas a eventos extremos (dirección del viento, velocidad del viento y contenido de humedad de los combustibles) y las coordenadas de las igniciones para los incendios que se desean simular. El archivo de paisaje es una retícula regular que contiene para cada pixel la información del terreno (pendiente, elevaciones y exposición), modelos de combustible y los parámetros de copas (altura de copas, densidad de copas, altura de la base de la copa y fracción de cabida cubierta). La información del terreno y los parámetros de copas se obtuvieron a partir de datos LiDAR (ign.es) y el mapa de modelos de combustible se generó mediante la asignación de modelos de combustible estándar (Fernandes, 2009; Scott y Burgan, 2005) a los diferentes usos de suelo, considerando como referencia los polígonos de SIGPAC 2017 (sigpac.navarra.es) y la información descriptiva (es decir, grado de cobertura, es- 
pecies arbustivas y las clases naturales de edad del estrato arbolado) extraída del mapa de usos de suelo y aprovechamientos de Navarra (año 2012; idena.navarra.es). Para la generación del archivo de paisaje con ArcFuels (Ager et al., 2011) se empleó la extensión del área de ocurrencia (36.000 ha; Fig. 2A) con el objeto de considerar la entrada de incendios iniciados fuera del área de estudio. Las condiciones meteorológicas extremas asociadas a los grandes incendios forestales se determinaron con Fire Family Plus (Bradshaw y McCormick, 2000), a partir de la serie histórica de datos horarios registrados en la estación automática de Pamplona durante los últimos 17 años (meteonavarra. es). Se consideró el percentil 97 como estadístico de referencia para fijar la velocidad del viento y el contenido de humedad (Nelson, 2000) en las simulaciones (Fig. 5). Se obtuvieron un total de cinco escenarios tipo, uno para cada dirección de viento (Alcasena et al., 2017) (Tabla 1). A partir del mapa de ocurrencia se generó un patrón de 10.000 igniciones, listado de igniciones que fue empleado posteriormente como dato de entrada en la simulación de incendios.

Tabla 1. Condiciones meteorológicas asociadas a los grandes incendios forestales en el área de estudio (Alcasena et al., 2017). Los datos meteorológicos fueron obtenidos de la estación automática de Pamplona (meteo.navarra.es) y el contenido de humedad fue derivado a partir del percentil 97 del ERC-G (Nelson, 2000). La velocidad del viento corresponde respectivamente al percentil 97 de cada dirección. Se emplearon modelos de combustible estándar (Fernandes, 2009;

Scott y Burgan, 2005), que fueron asignados a los diferentes usos de suelo (idena.navarra.es).

\begin{tabular}{|c|c|c|c|c|c|c|}
\hline \multicolumn{2}{|c|}{ Escenario de viento } & \multicolumn{4}{c|}{ Contenido de humedad (\%) } \\
\hline \multirow{2}{*}{$\begin{array}{c}\text { Dirección } \\
\text { (acimut) }\end{array}$} & $\begin{array}{c}\text { Velocidad } \\
\left(\mathbf{k m ~ h}^{-1}\right)\end{array}$ & Probabilidad & $\begin{array}{c}\text { Categoría de } \\
\text { combustible }\end{array}$ & $\begin{array}{c}\text { Modelos de combustible } \\
\text { GS1, GR5, } \\
\text { GR2, GR4, } \\
\text { SH6, SH5 }\end{array}$ & $\begin{array}{c}\text { TU3, PCL, } \\
\text { SH3, GR3 }\end{array}$ & $\begin{array}{c}\text { GR1, SH3, } \\
\text { TL2, SH8 }\end{array}$ \\
\hline 67,5 & 32 & 0,43 & $1-\mathrm{h}$ & 4 & 6 & 8 \\
\hline 337,5 & 35 & 0,28 & $10-\mathrm{h}$ & 5 & 7 & 9 \\
\hline 45 & 19 & 0,17 & $100-\mathrm{h}$ & 8 & 9 & 12 \\
\hline 180 & 31 & 0,06 & Vivo herbáceo & 20 & 45 & 70 \\
\hline 22,5 & 23 & 0,06 & Vivo leñoso & 60 & 85 & 100 \\
\hline
\end{tabular}

Se realizó una simulación con 10.000 igniciones por cada uno de los 5 escenarios (50.000 incendios en total) con el algoritmo "minimum travel time" (MTT) (Finney, 2002) implementado en el simulador FlamMap (Finney, 2006). Debido a que FlamMap MTT simula todos los incendios con las mismas condiciones meteorológicas (dirección de viento, velocidad de viento y contenido de humedad del combustible constante), se realizó una simulación para cada escenario de viento. El resultado final fue obtenido combinando los resultados de los diferentes escenarios, considerando la probabilidad de cada escenario (Tabla 1). Se fijó una duración en las simulaciones de 6 horas, duración del periodo de propagación activa observado en los grandes incendios históricos en el área de estudio (incendio de Juslapeña en 2009). Todos los píxeles se quemaron al menos una vez y más de 100 veces en promedio. Las simulaciones se 
realizaron a $30 \mathrm{~m}$ de resolución y en propagación libre, sin fijar barreras ni efectos de contención ya que la propagación del frente se considera resistente a los medios de extinción bajo condiciones meteorológicas extremas. Se obtuvieron resultados de salida de probabilidad de quema, intensidad del fuego y perímetros que se detallan a continuación.

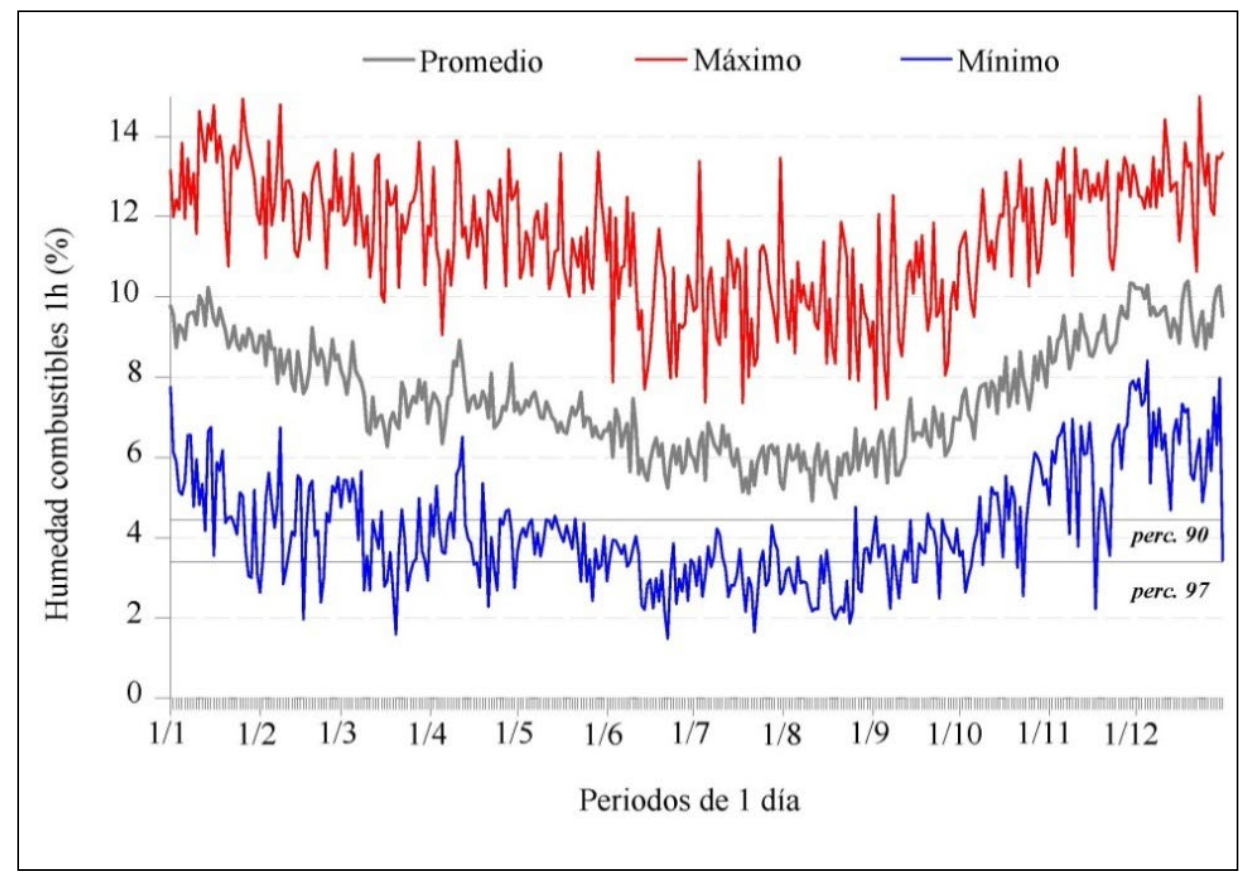

Figura 5. Evolución interanual en el contenido de humedad del combustible fino de $1 \mathrm{~h}$ de tiempo de retardo en el área de estudio. Los valores fueron calculados (Nelson, 2000) a partir de los datos horarios de precipitación, humedad relativa, temperatura, vientos y radiación solar (1997 a 2015) registrados en la estación automática de la Universidad Pública de Navarra (Pamplona,

Navarra). Para la simulación de incendios en el área de estudio se emplearon los valores extremos correspondientes al percentil 97.

La probabilidad de quema o "burn probability" $(B P)$ es una trama regular con valores entre 0 y 1 , obtenido a partir de la relación entre el número de veces que se quema cada píxel y el número de igniciones empleadas en la simulación. El resultado de BP obtenido en este estudio es un valor de probabilidad condicionado a la ocurrencia de un gran incendio bajo condiciones meteorológicas extremas previamente detalladas (Tabla 1). Además, FlamMap calcula en cada incendio la longitud de llama para cada pixel. Posteriormente, después de simular independientemente todos los incendios a partir de las igniciones indicadas, en cada píxel la intensidad se obtiene como un valor de probabilidad o "flame length probability" (FLP) para cada uno de los 20 niveles o "fire intensity levels" (FILs) de 0,5 m de longitud de llama, siendo para la clase 20 la longitud de llama $>9,5 \mathrm{~m}\left(\mathrm{FIL}_{1}=0-0,5\right.$ hasta $\left.\mathrm{FIL}_{20}>9,5 \mathrm{~m}\right)$. De este modo, la intensi- 
dad en cada pixel considera la dirección en la propagación del frente (es decir, cabeza, cola y flanco). La suma de lodos los FLP en los 20 niveles es de 1 en cada pixel. Finalmente, también se obtuvieron los polígonos de los perímetros para cada uno de los incendios simulados. Cada perímetro tiene asignado el valor de la superficie quemada y las coordenadas de su ignición. Se obtuvieron un total de 50.000 perímetros, 10.000 por cada escenario. Para los análisis posteriores únicamente se emplearon los perímetros de los grandes incendios ( $>100$ ha) ya que estos son responsables de la mayor parte del área quemada.

\subsection{Efectos del fuego}

Los efectos del fuego se pueden cuantificar empleando relaciones de susceptibilidad derivadas del criterio experto o a partir de modelos de mortalidad en el caso de las especies arboladas. En ambos casos, los efectos del fuego (pérdidas o beneficios) se determinan para diferentes niveles de intensidad (principal factor causativo de riesgo) (Scott et al., 2013). La aproximación de los efectos esperados a diferentes niveles de intensidad nos permite integrarlos con los resultados obtenidos en la simulación (probabilidad de quema e intensidad del fuego) y evaluar así el riesgo (Ager et al., 2011). En este estudio se emplearon funciones de respuesta o "response functions" (RFs) (Thompson et al., 2011) para determinar los efectos del fuego en viviendas residenciales. Las pérdidas en las viviendas se establecieron como un porcentaje de su valor total y se determinaron con el método Delphi (Dalkey y Helmer, 1963). El método Delphi es una técnica de comunicación estructurada desarrollada como un método sistemático e interactivo de predicción y basada en un panel de expertos (Lovreglio et al., 2010; Meddour-Sahar et al., 2013). Los resultados fueron obtenidos de encuestas realizadas a responsables de Bomberos de Navarra con experiencia en las labores de extinción de incendios de interfaz urbano forestal en el área de estudio, encuestas en las que se les solicitó que indicasen las pérdidas esperadas como un porcentaje del valor de las viviendas afectadas (de $0 \%$ sin daños, a $-100 \%$ para la destrucción total de la estructura) a diferentes niveles de intensidad (Alcasena et al., 2017) (Tabla 2). La inmensa mayoría de las estructuras en el área de estudio presentan muros de carga de piedra o ladrillo con revestimiento exterior, disponen de persianas (de madera, aluminio o plástico) y las cubiertas son de madera en la mayoría de los casos. Las encuestas se realizaron de modo anónimo en dos etapas, en la primera se asignaron los valores y en la segunda se afinaron los resultados. Por otro lado, con el objeto de determinar los efectos del fuego en las repoblaciones de pino laricio, se empleó un modelo de mortalidad genérico desarrollado para coníferas que considera variables como el espesor de la corteza y altura de copa (Fernandes et al., 2008; Peterson y Ryan, 1986). A partir de datos de inventario disponibles por el Guarderío Forestal se calculó la mortalidad media esperada a nivel de rodal para diferentes niveles de intensidad (Alcasena et al., 2016a) (Tabla 2). 
Tabla 2. Funciones de respuesta $(R F)$ para viviendas residenciales (pérdidas con respecto a su valor, en \%) y mortalidad (valor medio, en \%) post-incendio en repoblaciones de pino laricio para diferentes niveles de intensidad (Alcasena et al., 2017; Alcasena et al., 2016a). Los niveles de intensidad (FIL) se especifican para rangos de 0,5 m de longitud de llama. Por encima de

$2,5 m$ de longitud de llama la mortalidad en los pinares es del $100 \%$.

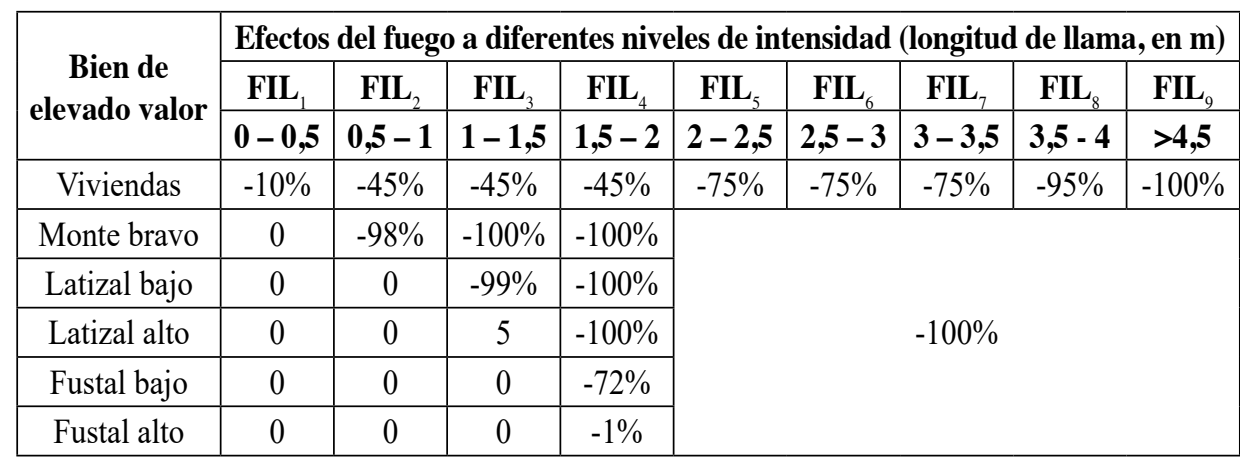

\subsection{Riesgo de incendios}

El riesgo de incendios (Finney, 2005; Scott et al., 2013) se cuantificó como la expectativa de pérdida económica $(e E L)$ en caso de gran incendio, mediante el empleo de la siguiente ecuación:

$$
e E L=V \times \sum_{i=1}^{20} B P \times F L P_{i} \times R F_{i}
$$

donde $B P$ es la probabilidad de quema (resultado de simulación), $F L P_{\mathrm{i}}$ es la probabilidad de un fuego para el nivel de intensidad $i$ (resultado de simulación), $R F_{\mathrm{i}}$ es la función de respuesta para el nivel de intensidad $i$ y $V$ es el valor económico del bien afectado por el fuego (es decir, repoblaciones y viviendas residenciales). Los valores económicos de la madera para las diferentes clases de edad ( $€ \times \mathrm{m}^{3}$ con corteza en pie) se obtuvieron a partir de los precios medios de adjudicación definitiva alcanzados en las subastas realizadas en el área de estudio (Ayuntamiento de Juslapeña, com. pers. 2014). Se consideraron diferentes valores en base a las dimensiones de los productos y su destino (trituración, embalaje o sierra). Para las viviendas residenciales se emplearon los valores indicativos a efectos de Impuestos de Transmisiones Patrimoniales y Actos Jurídicos Documentados, obtenidos a partir de datos catastrales (catastro.navarra.es; Decreto Foral 334/2001 de 26 de noviembre). En el caso de las repoblaciones se generó una cartografía de riesgo con valores de pérdidas económicas esperadas en los rodales a $30 \mathrm{~m}$ de resolución, considerando las existencias $\left(\mathrm{m}^{3}\right.$ con corteza ha $\left.{ }^{-1}\right)$ para cuantificar las pérdidas totales. Para las viviendas individuales se consideró el valor medio de $e E L$ en todos los píxeles dentro de la zona de ignición en viviendas (buffer de 60 metros) (Cohen, 2008). 


\subsection{Optimización espacial de tratamientos}

Para la optimización espacial de los tratamientos y la identificación de los polígonos o rodales a tratar preferentemente se empleó el programa Landscape Treatment Designer (LTD) (Ager et al., 2016b). Este programa permite configurar el mosaico de tratamientos que maximiza la contribución del área tratada con respecto a uno o varios objetivos, para satisfacer, en medida de lo posible, las necesidades de los diferentes agentes sociales (es decir, soluciones multiobjetivo). En este estudio se establecieron tres objetivos para los tratamientos: (1) la reducción del riesgo de incendios en masas arboladas (pinares y robledales), (2) la reducción del riesgo de incendios en núcleos urbanos, y (3) la obtención de leñas o madera en el tratamiento de combustibles. A cada uno de los objetivos se le asocia un parámetro cuantitativo que permita captar el gradiente espacial existente en el territorio e identificar así los rodales o polígonos estratégicos con valores más elevados. En cada parcela o rodal a tratar se asume un grado de cumplimiento para cada objetivo proporcional al valor cuantitativo del parámetro asociado (es decir, mayor grado de cumplimiento de los objetivos en rodales con valores más elevados) con respecto al total en el área de estudio.

a) Parámetros cuantitativos asignados a los objetivos

Con la finalidad de identificar las masas arboladas (pinares y robledales) que presentan unas mayores pérdidas potenciales en caso de gran incendio forestal se empleó el parámetro de probabilidad de quema en alta intensidad o "high intensity burn probability" (HIBP) (Lozano et al., 2017) , que fue calculado mediante la siguiente ecuación:

$$
H I B P=\sum_{i=6}^{20} F L P_{i} \times B P
$$

El valor de HIBP se calculó para cada píxel a 30 m de resolución empleando los resultados de probabilidad e intensidad de las simulaciones. Por encima de 2,5 m de longitud de llama en fuegos de superficie ( $\geq \mathrm{FLP}_{6 ;}$ Tabla 2 ) la mortalidad es muy elevada o total.

Para la reducción del riesgo de incendios en la interfaz urbano-forestal, se empleó la medida de transmisión como referencia para identificar los polígonos o rodales en el paisaje que son el origen de incendios que alcanzan un gran número de viviendas en los núcleos urbanos del área de estudio. Los perímetros (resultados de simulación) se intersectaron con los centroides de las viviendas residenciales (catastro.navarra.es) y el número de viviendas intersectadas fue respectivamente asignado a las coordenadas de cada ignición. A continuación se calculó la transmisión $(T F)$ para cada polígono de terreno forestal con la siguiente ecuación (Alcasena et al., 2017):

$$
T F_{i j}=\frac{R H_{j}}{N_{i}}
$$

donde $R H$ son el número de viviendas residenciales afectadas en $j$ (Valle de Juslapeña) y $N$ es el número de igniciones dentro del polígono de terreno forestal $i$ considerado en el análisis. En este análisis también se consideró la transmisión de incendios iniciados dentro del área de estudio (es decir, todos los $i$ polígonos o rodales dentro de $j$ ).

Debido a que actualmente en el área de estudio no existen planes de ordenación ni datos de inventario detallados a nivel de rodal, son las medidas de espesura el criterio que habitualmente se considera para determinar la necesidad de ejecución de claras. A falta 
de datos de inventario detallados a nivel de rodal (en todas las masas, robledales y pinares) que nos pudiesen facilitar el cálculo de índices de espesura (por ej., Hart-Becking o Stand Density Index) se empleó el valor de la fracción de cabida cubierta (\%) como una primera aproximación (FCC), ya que en última instancia el objetivo en el proceso de optimización es priorizar tratamientos y no cuantificar las extracciones. La fracción de cabida cubierta fue obtenida en alta resolución a partir de la nube de puntos LiDAR con FUSION (McGaughey, 2018). La altura de referencia considerada para diferenciar el estrato arbolado y el estrato arbustivo se fijó en $3 \mathrm{~m}$. La FCC se obtuvo a partir de la relación entre el número de primeros retornos sobre los $3 \mathrm{~m}$ y el número total de primeros retornos en píxeles de $30 \mathrm{~m}$ de resolución.

b) Área de planificación y unidades de tratamiento

Para delimitar la extensión del área de planificación donde se pretende priorizar el tratamiento de combustibles a escala de paisaje, en este estudio se consideró la cuenca de exposición a incendios o "fireshed" de las viviendas residenciales en Valle de Juslapeña y no el límite administrativo. La delimitación de la cuenca de exposición se realizó a partir de una trama continua de transmisión, generada mediante una interpolación espacial a partir de los valores asignados a cada ignición. Concretamente, la trama se generó a 30 m de resolución con sistemas de información geográfica (SIG) mediante una interpolación bilineal y el límite de la cuenca se fijó en los píxeles con valores de transmisión igual a 0 . A continuación, para delimitar los rodales (es decir, unidades de tratamiento de combustibles) se consideraron los polígonos de terreno forestal y pasto arbolado de SIGPAC 2017 (e: 1/5000; sigpac.navarra.es) situados dentro de la cuenca de exposición. No obstante, los polígonos con una superficie superior a 10 ha fueron divididos en rodales con una superficie máxima de 5 ha considerando pistas forestales, cursos de aguas superficiales, divisorias de aguas y cambios bruscos de pendiente. A continuación, a cada polígono dentro de la cuenca de afectación $(n=7218$ polígonos, 9880 ha de superficie) se le asignó la suma del valor de todos los píxeles para cada objetivo (HIBP, FCC y TF). Para poder normalizar los valores de cada objetivo en cada polígono, a cada polígono se le asignó la contribución (\%) del valor con respecto a la suma total en todos los polígonos (por ej., si la suma de HIBP en todos los polígonos fue de 3140, al polígono con un HIBP de 7,85 le corresponde el 0,25\%).

c) Maximización de objetivos

Se empleó la siguiente ecuación para la maximización de objetivos y la identificación de los rodales a tratar (Ager et al., 2016b):

$$
\operatorname{Max} \sum_{j=1}^{k}\left(Z_{j} \times \sum W_{i} N_{i j}\right)
$$

condicionado a

$$
\sum_{j=1}^{k}\left(Z_{j} A_{j}\right) \leq C
$$

donde $\mathrm{C}$ es el factor limitante (es decir, área de terreno a tratar), $\mathrm{Z}$ es un vector binario para indicar si el rodal $j$ se trata o no (por ej., $Z_{\mathrm{j}}=1$ para rodales tratados y 0 para rodales no tratados), $N_{\mathrm{ij}}$ es la contribución para el objetivo $i$ en el rodal $j$ si es tratado, $A$ es el área del rodal $j$ si es tratado y $W$ es un coeficiente para asignar pesos que per- 
mite enfatizar un objetivo respecto a otro. Cuando todos los objetivos tienen el mismo peso (W) se asigna el valor de 1 a todos ellos. El área a tratar se fijó en un $20 \%$ de la superficie forestal arbolada ( $\mathrm{A}=1976$ ha, $\sim 10 \%$ de la cuenca de exposición). Estudios previos indican que las bajas intervenciones (área tratada $<18-20 \%$ ) no tienen efectos sustanciales en el comportamiento y la propagación de los grandes incendios (Ager et al., 2013; Finney et al., 2006). Para trazar las fronteras de posibilidad de producción a modo de proyección tridimensional (óptimo alcanzable para todas las combinaciones posibles en los tres objetivos) se emplearon los 415 puntos correspondientes a las combinaciones de pesos (W) entre 0 y 7 para incrementos de 1 unidad (LTD permite asignar pesos a objetivos en base a las preferencias de los gestores). Por ejemplo, un peso de 1 para FCC y 0 para HIBP y TF identifica los polígonos donde la suma de la contribución (\%) en FCC sea máxima, independientemente de los valores de HIBP y TF. En las parcelas a tratar se contempla la combinación de varias técnicas (es decir, quemas prescritas, desbroces y claras) que deberán en cada caso adaptarse a los condicionantes socioeconómicos y topográficos existentes en las parcelas. En el proceso de optimización se excluyeron las parcelas con longitudes de llama inferiores a 1,2 m puesto que la gestión de combustibles predominantemente herbáceos se realiza mediante la ganadería extensiva en el área de estudio.

\section{Resultados}

\subsection{Riesgo de incendios}

La expectativa de pérdidas económicas en caso de gran incendio (eEL) presentó una gran variabilidad en el área de estudio (Fig. 6). Tal y como era de esperar, los daños en las viviendas residenciales fueron superiores a los daños en las repoblaciones de carácter productor. Las mayores pérdidas se obtuvieron en las viviendas de las entidades locales situadas al sur $\left(>8000 €\right.$ vivienda $\left.^{-1}\right)$, siendo las pérdidas hasta más de ocho veces superiores con respecto a las localidades del norte (Fig. 6B). El valor medio en el área de estudio fue de $7.955 €$ vivienda $^{-1}$. La mayor probabilidad de quema (BP) obtenida al sur del área de estudio resultó el factor más decisivo en la expectativa de pérdida económica (eEL) ya que la variabilidad en las valoraciones económicas es reducida. La mayoría de viviendas (>75\%) presentaban valores de entre los 100.000 y $200.000 €$, rara vez se superan los $300.000 €$. En las repoblaciones las pérdidas más elevadas se localizan en la parte central $\left(>500 €\right.$ ha $^{-1}$; Fig. $6 \mathrm{~A})$, correspondiendo con los rodales en edad de latizal alto y fustal bajo capitalizados en existencias y con exposiciones elevadas (elevada probabilidad de quema y elevadas intensidades). Los pinares en edad de fustal alto localizados en áreas remotas presentaron pérdidas potenciales muy reducidas. En las repoblaciones, la variabilidad en las existencias y el valor de la madera resultaron factores decisivos que establecieron grandes diferencias entre los resultados de los diferentes rodales. El precio de la madera puede incrementarse hasta en más de ocho veces, puesto que oscila entre los $<3 € \mathrm{~m}^{3}$ con corteza (trituración, pasta de papel) y los $>25 € \mathrm{~m}^{3}$ con corteza (madera de sierra). 


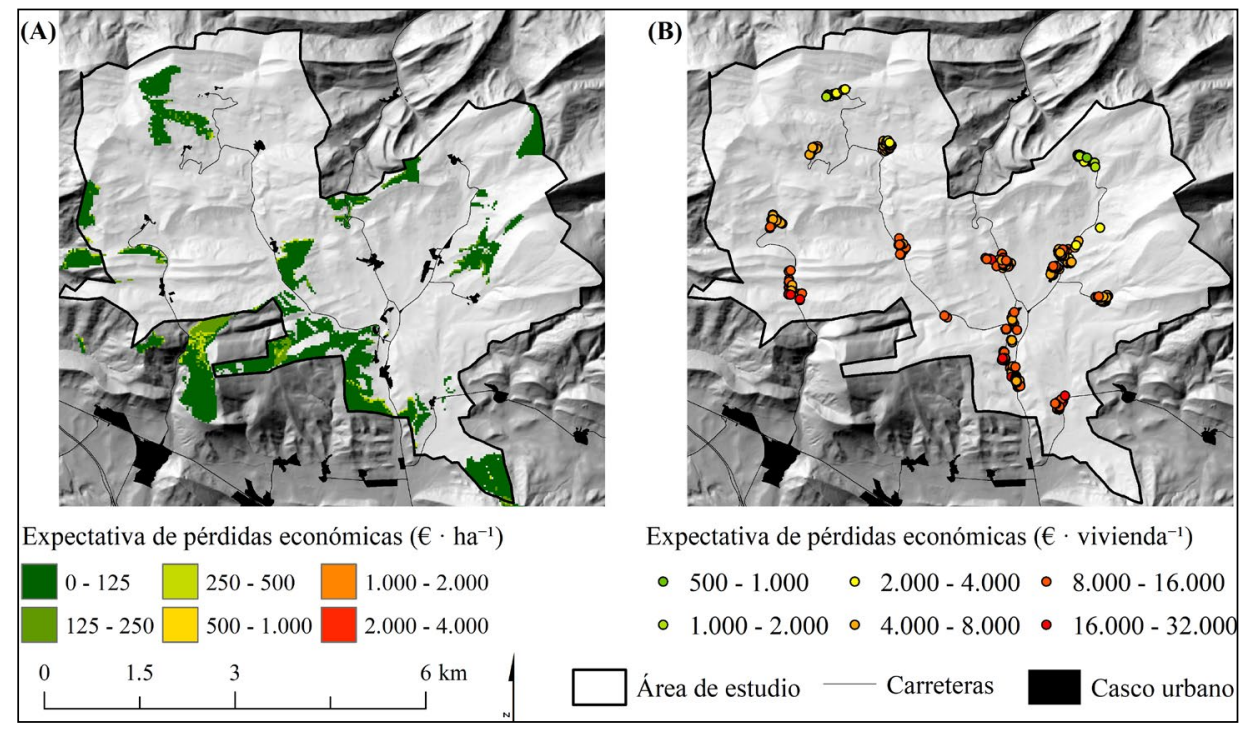

Figura 6. Mapa de riesgo para las repoblaciones de pino laricio (A) y viviendas $(B)$ en el área de estudio. Para la valoración de las pérdidas en las repoblaciones se consideró la mortalidad esperada (\%), existencias y los precios de la madera alcanzados en subastas.

Las viviendas (puntos) se agrupan en núcleos urbanos. En el caso de las viviendas se consideraron funciones de respuesta (pérdidas, en \%) y los valores derivados de catastro para las edificaciones. Los resultados que se presentan están condicionados a la ocurrencia de un gran incendio forestal.

\subsection{Objetivos de los tratamientos}

Para poder asignar los valores correspondientes a los tres objetivos en cada rodal, primero se calcularon los valores de HIBP y TF a $30 \mathrm{~m}$ de resolución en el paisaje de 36.000 ha considerado en la simulación. El mapa de probabilidad de quema en alta intensidad (HIBP) alcanzó los valores más elevados en los fondos de valle de la parte central del área de estudio (Fig. 7A). En las montañas, exposiciones norte y cotas más elevadas los valores fueron bajos $(<0,03)$ debido a la baja probabilidad de quema (BP) y bajas intensidades (FIL). Los resultados de HIBP fueron especialmente bajos en los hayedos limítrofes localizados en la zona norte, que representan una barrera a la propagación de los incendios. Los valores de transmisión (TF) fueron especialmente elevados para todo el fondo de valle en el área de estudio (>200 viviendas residenciales) (Fig. 7B). La cuenca de exposición a incendios (contorno delimitado por $\mathrm{TF}=0$; Fig. 7B) no se corresponde con el límite administrativo del Valle de Juslapeña y los incendios iniciados fuera del área de estudio (incluso a más de $5 \mathrm{~km}$ de distancia) pueden representar un grave riesgo. Cabe destacar el gran potencial destructor de los incendios iniciados sobre la llanura (campos de cereal) situada en los términos municipales al sur del área de estudio. 


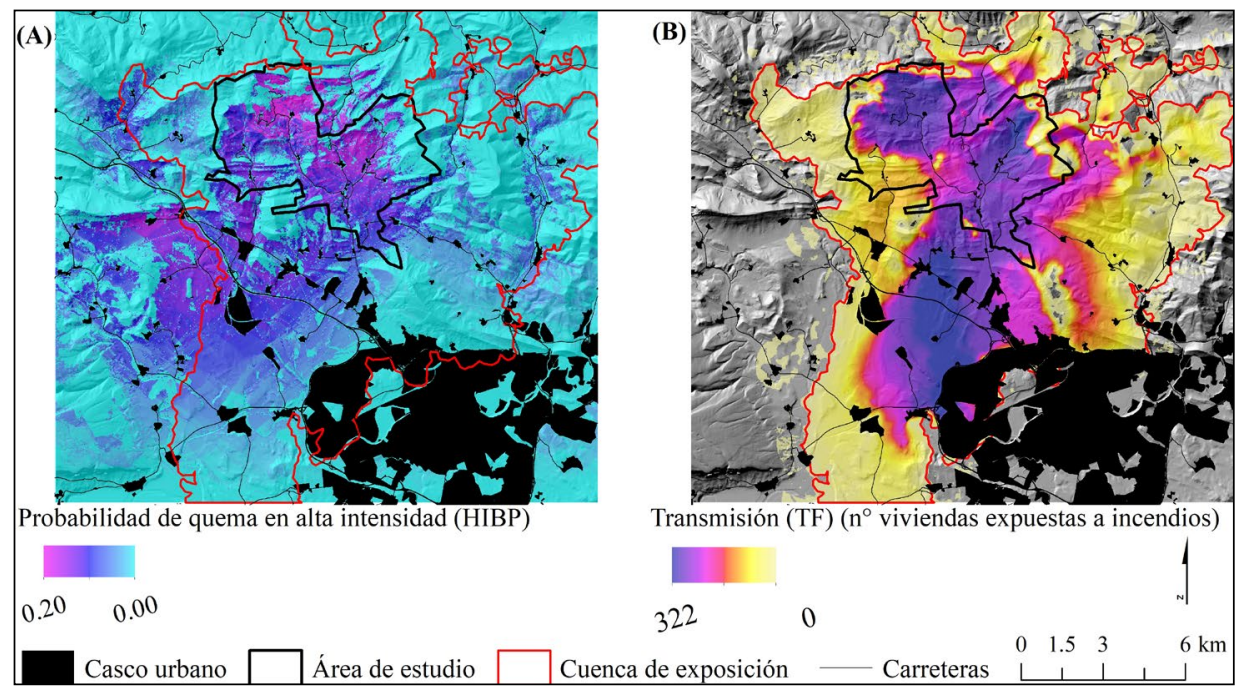

Figura 7. Mapa de probabilidad de quema en alta intensidad (HIBP; $>2,5 \mathrm{~m}$ de longitud de llama) (A) y transmisión de incendios a viviendas (número de viviendas expuestas a incendios) $(B)$ en alta resolución $(30 \mathrm{~m})$. Los resultados fueron obtenidos para condiciones meteorológicas extremas (percentil 97) y una duración de 6 h en propagación libre. La HIBP está condicionada a la ocurrencia de un gran incendio forestal. El contorno exterior del mapa de transmisión delimita la cuenca de exposición a incendios o "fireshed" y fue empleada para acotar la superficie (polígonos) a considerar en la optimización espacial.

\subsection{Frontera de posibilidades de producción}

El proceso de optimización nos permitió maximizar la contribución de los polígonos tratados ( $20 \%$ de la superficie forestal dentro de la cuenca de exposición) para los tres objetivos (Fig. 8). Los resultados correspondientes al límite de las fronteras de posibilidades de producción fueron representados a modo de proyección tridimensional. La proyección representa el óptimo y la máxima contribución posible, las combinaciones por encima de la superficie son inalcanzables y las situadas bajo la proyección suponen combinaciones ineficientes. Las contribuciones de HIBP, FCC y TF presentaron variaciones entre $21-55 \%, 15-33 \%$ y $15-39 \%$ respectivamente. El valor máximo alcanzable en cada objetivo se obtuvo cuando toda la superficie tratada se destinó a maximizar un único objetivo. Dependiendo de las prioridades establecidas por los gestores del territorio (es decir, pesos asignados a los objetivos) y las limitaciones económicas (necesidades de costear los tratamientos con las extracciones), la suma de la contribución de todos los polígonos tratados se localizaría en uno u otro punto de la proyección tridimensional. Para situaciones en las que las limitaciones económicas sean un factor determinante en el momento de ejecutar los trabajos, la obtención de beneficios puede ser decisiva y se buscará maximizar la contribución de FCC. En este caso la combinación de la suma en todas las contribuciones se encontrará localizada en la zona de colores más cálidos donde las masas tratadas presentan una mayor espesura y las extracciones en las claras serán 
mayores. Si por el contrario no existen grandes limitaciones económicas, los tratamientos buscarán maximizar HIBP y TF (zona de colores fríos).

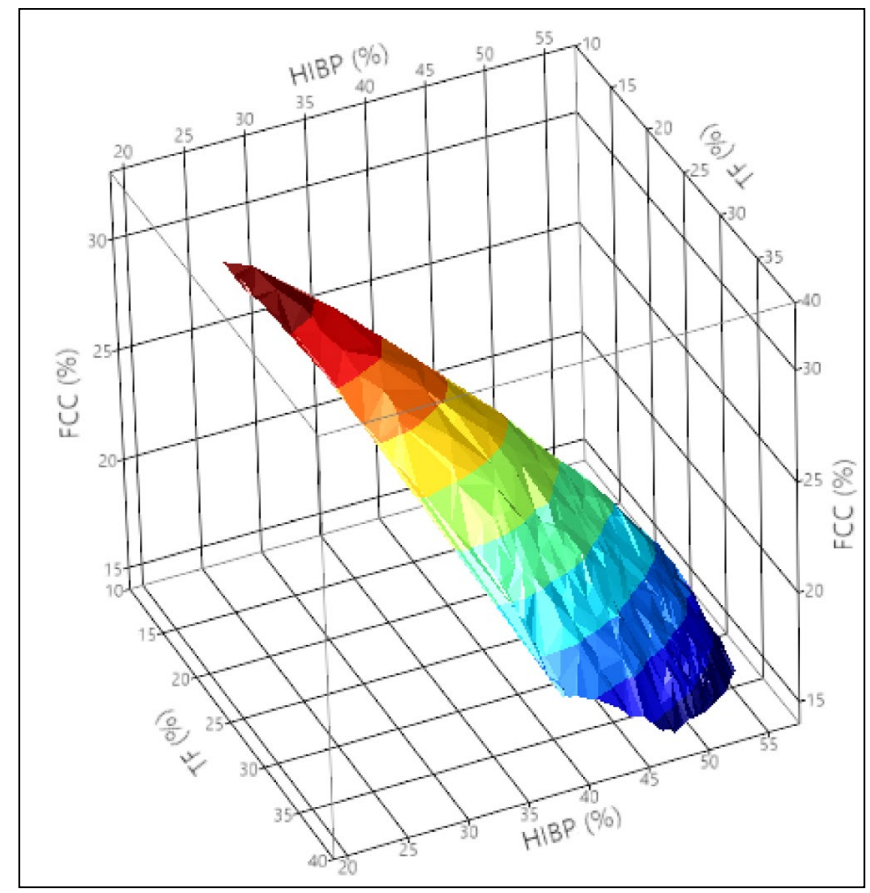

Figura 8. Fronteras de posibilidades de producción para la transmisión de incendios a viviendas residenciales $(T F)$, probabilidad de quema en alta intensidad (HIBP) y fracción de cabida cubierta (FCC). La superficie tridimensional proyectada indica el máximo alcance posible (\%) y óptimo para los tres objetivos al efectuar tratamientos en un $20 \%$ de la superficie forestal arbolada. Las soluciones sobre la proyección son inalcanzables con los recursos disponibles y las situadas bajo la proyección son ineficientes.

\subsection{Localización de los tratamientos}

Se generaron mapas para la maximización de los diferentes objetivos por separado (empleando pesos de $\mathrm{W}=1$ para el objetivo a maximizar, y $\mathrm{W}=0$ para el resto), en los que se identificó el mosaico de rodales a tratar para cada uno de ellos (Fig. 9). En el caso de los tratamientos encaminados a reducir la transmisión de incendios a viviendas, la mayoría de los rodales seleccionados se concentraron dentro del área de estudio y en un entorno próximo. En cuanto a las masas arboladas con una elevada exposición a incendios, los rodales identificados se correspondieron principalmente con repoblaciones de pino laricio y robledales procedentes de regeneración natural en edad de latizal. Son masas próximas a los cultivos de cereal donde los incendios que se transmiten y propagan por el fondo de valle impactan con las zonas forestales arboladas. Los rodales con una mayor espesura donde a priori las extracciones de leñas y madera serían más elevadas, 
corresponden a todo tipo de masas arboladas dispersas dentro de la cuenca de afectación. Estas masas corresponden principalmente a pinares en los que han transcurrido más de 15 años desde la clara anterior y robledales o hayedos que presentan un difícil acceso y con un aprovechamiento de leñas para hogares complicado (es decir, elevada pendiente $\mathrm{y}$ ausencia de pistas forestales).

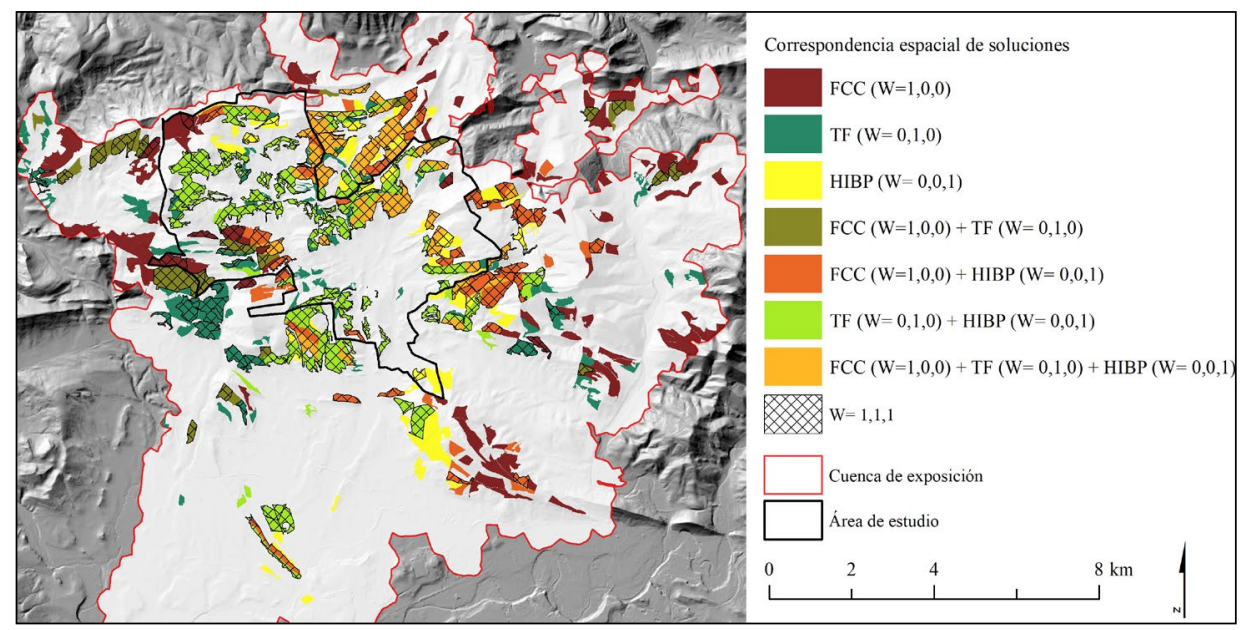

Figura 9. Correspondencia espacial de las parcelas o rodales a tratar, identificados a partir de la maximización de los tres objetivos por separado: la reducción de riesgo de incendios en viviendas $(T F)$, mitigación de daños en masas arboladas con una elevada exposición (HIBP) y obtención de lañas o madera (FCC). Además, también se presenta la solución correspondiente al mismo peso o prioridad a todos los objetivos $(W=1,1,1)$, que se indica con una trama cuadriculada. Los resultados fueron obtenidos para tratamientos del $20 \%$ del terreno forestal dentro de la cuenca de exposición por incendios a núcleos urbanos. Únicamente se consideraron en los tratamientos los polígonos de terreno forestal (no pastizales) con intensidades medias superiores a 1,2 m de longitud de llama.

Con el objeto de conocer la correspondencia espacial de tratamientos para los tres objetivos maximizados por separado, se superpusieron los rodales estratégicos identificados en las tres soluciones. Además, se empleó el LTD para la identificación de los rodales a tratar cuando la prioridad (o peso) en los 3 objetivos es la misma ( $\mathrm{W}=1,1,1)$. Nótese que los rodales seleccionados a partir de la superposición de los tres objetivos maximizados por separado no se corresponden con la combinación de pesos $\mathrm{W}=1,1$, 1 (trama cuadriculada) y sus contribuciones se situarían bajo el plano de la frontera de posibilidad de producción (Fig. 9). No obstante, la correspondencia espacial puede ser empleada para establecer el orden de ejecución en los tratamientos (es decir, ejecutar los tratamientos primero en rodales con mayor correspondencia de objetivos maximizados independientemente), ya que los proyectos de tratamiento de combustibles requieren varios años para completar su ejecución. En general, la mayoría de los rodales a tratar seleccionados en los tres objetivos se encuentran situados al límite norte ya que toda la parte sur de la cuenca de afectación se corresponde con campos de cultivo de cereal que han sido excluidos del análisis. 


\section{Discusión}

La expansión de la superficie forestal arbolada durante las últimas seis décadas ha desencadenado un régimen de incendios forestales resistente a la extinción. Esta nueva superficie es un bosque no gestionado con un gran número de pies en las clases diamétricas inferiores, caracterizado por una elevada carga y continuidad de combustibles. En las regiones Mediterráneas, este cambio ha supuesto la desaparición del paisaje cultural en mosaico adaptado a un régimen de incendios recurrentes de baja severidad (Cervera et al., 2016). A pesar de que multitud de estudios enfatizan la necesidad de reducir los combustibles forestales para combatir a los grandes incendios forestales (Bovio et al., 2017; Fernandes, 2013; Madrigal et al., 2016), los servicios de prevención no disponen de las herramientas necesarias para optimizar los recursos económicos disponibles.

Actualmente la localización de tratamientos preventivos se determina en base al criterio experto. Sin embargo, la ausencia de una metodología técnicamente consistente aceptada por los gestores del territorio dificulta el establecimiento de un procedimiento estandarizado que facilite su diseño e implementación. El criterio experto se basa en el estudio del comportamiento y la propagación de incendios históricos para determinar la localización de puntos estratégicos a escala de paisaje o macizo forestal, además de considerar la tipología de combustibles forestales (es decir, carga y estructura) en la prescripción del tipo de tratamiento más adecuado en cada caso (por ej., clara, quema, desbroce o poda). No obstante, en ocasiones, los tratamientos son sistemáticos y consisten en la apertura o mantenimiento de redes de cortafuegos que siguen principalmente divisorias de cuencas hidrográficas para segmentar el territorio en bloques o áreas de gestión. Las principales limitaciones del criterio experto son la imposibilidad de evaluar cuantitativamente el efecto de las diferentes alternativas en la reducción del riesgo de incendios (por ej., variación de la forma y superficie de parcelas tratadas, además del tipo de tratamiento empleado), así como la dificultad de establecer prioridades entre parcelas a tratar situadas en territorios extensos $\left(>10.000 \mathrm{~km}^{2}\right)$ con un régimen de incendios cambiante. Nosotros proponemos una metodología que permite determinar dónde se concentran las pérdidas más elevadas, además de facilitar el diseño estratégico de tratamientos preventivos en paisajes multifuncionales. La disposición espacial de tratamientos contempla por tanto una solución integral con dos estrategias complementarias diferentes (Calkin et al., 2014; Penman et al., 2015): tratamientos junto a los bienes con elevadas expectativas de pérdida económica y tratamientos multifuncionales a escala de paisaje. Los tratamientos en el entorno próximo a viviendas residenciales y pinares de carácter productor disminuyen notablemente el riesgo en la parcela o rodal tratado y se debe a la reducción en la intensidad del fuego (es decir, longitud de llama). En el segundo caso, el mosaico de tratamientos estratégicos pretende reducir la transmisión a núcleos urbanos (es decir, probabilidad de quema) así como la exposición en masas arboladas, además de maximizar las extracciones de leña o madera.

Este estudio supone una primera aproximación que contribuye a esclarecer la complejidad existente en los procesos de optimización espacial. En líneas generales, la optimización en el tratamiento de combustibles se emplea para determinar dónde se deben localizar los tratamientos y cuánta superficie de terreno se requiere tratar, de modo tal 
que las inversiones realizadas en los tratamientos minimicen al máximo las pérdidas asociadas a incendios forestales (Finney, 2007; Finney et al., 2007). A su vez, la variabilidad en la intensidad de los tratamientos (es decir, cantidad de biomasa retirada y estructura en los combustibles tratados) y las diferencias existentes en el tamaño, forma y grado de agregación de las parcelas incrementa sustancialmente la complejidad del proceso (Arca et al., 2015; Finney, 2004; Salis et al., 2018; Salis et al., 2016b; Scott et $a l .$, 2016). Del mismo modo, la existencia de condicionantes económicos (presupuestos limitados), medioambientales (normativa restrictiva en hábitats protegidos) y de propiedad o tenencia de la tierra (público o privada) hacen que la solución sea distinta en cada caso, siendo difícil la obtención de conclusiones generales. Con el objeto de aportar una solución consistente para el área de estudio, en este artículo se identifica el conjunto de rodales (mosaico de parcelas) a tratar de manera preferente (es decir, parcelas con los valores más elevados por unidad de superficie asociados a uno o varios objetivos). Para facilitar el cálculo se establecieron una serie de consideraciones generales, así como la exclusión de cultivos agrícolas (tierra arable) y pastos, el establecimiento de una superficie máxima a tratar como único factor condicionante y el empleo de la delimitación de las parcelas o rodales en base a catastro actual. Además, el hecho de acotar el "área tratable" empleando la cuenca de exposición a viviendas nos permitió "concentrar los tratamientos" en un área menor y excluir así las áreas más remotas.

Los incendios no entienden de límites administrativos y la responsabilidad en la gestión de combustibles es compartida y debe descomponerse en varias escalas (Calkin et al., 2011; Palaiologou et al., 2018). En los núcleos urbanos, los propietarios particulares de viviendas (y parcelas) y la administración local deben coordinarse e implicarse en el mantenimiento de los combustibles en zonas de interfaz urbano-forestal con el objeto de generar comunidades adaptadas a incendios forestales. En el área de estudio, el arado de los campos de cereal próximos a los núcleos urbanos (hasta $60 \mathrm{~m}$ ) inmediatamente después de la cosecha con un pase de cultivador, así como la conservación de zonas ajardinadas (es decir, franjas perimetrales) y huertas con una baja carga de combustibles reduciría significativamente la intensidad del frente del incendio en caso de impactar directamente con el núcleo urbano habitado (Alcasena et al., 2015). En el entorno más próximo a las viviendas $(<30 \mathrm{~m})$ el empleo de especies poco inflamables y la retirada de todo el combustible muerto en setos y jardines (por ej., hojarasca y restos de podas, incluso la leña apilada junto a viviendas o tanques de propano) pueden resultar determinantes para evitar la ignición de las estructuras y frenar la transmisión del fuego entre viviendas vecinas (Cohen, 2008). Además, tal y como demuestra la discordancia entre la cuenca de exposición (es decir, área de planificación de tratamientos) y el límite administrativo (Fig. 7), la colaboración entre municipios vecinos también resulta necesaria ya que los grandes incendios originados fuera del área de estudio pueden llegar a causar graves daños en los núcleos urbanos situados dentro del área de estudio (Alcasena et al., 2017). El desarrollo y la aplicación de normativa autonómica y ordenanzas municipales específicas pueden resultar de gran utilidad a tal efecto, requiriendo el cumplimiento de medidas preventivas.

Además de la gestión de combustibles en los núcleos urbanos, la reducción de la susceptibilidad al fuego en estructuras y la aplicación de medidas de autoprotección 
también contribuyen a reducir el riesgo. A pesar de que la ignición de las viviendas en esta tipología de interfaz urbano-forestal es principalmente causada por pavesas (es decir, el impacto directo del frente a elevadas intensidades contra las estructura es poco probable), el empleo de barnices o pinturas intumescentes en las estructuras y carpintería exterior, así como la instalación de persianas ignífugas aumentaría notablemente la resistencia de las estructuras al impacto directo del fuego (es decir, menores pérdidas en las FR para los mismos FIL). Además, las medidas de autoprotección consistentes en la habilitación de zonas seguras y la construcción de puntos de agua facilitan la operatividad y autonomía de los medios de extinción en los núcleos urbanos (Butler, 2014; Syphard et al., 2014). A su vez, la habilitación de centros de reunión seguros para el confinamiento de personas vulnerables (niños, mayores y personas con movilidad reducida) puede resultar una medida acertada en núcleos rurales con mala comunicación. Ante la imposibilidad de confinamiento resultaría necesario identificar cuáles son las vías de evacuación preferente en caso de emergencia para evitar accidentes. En el área de estudio los incendios iniciados en el fondo de valle y que se propagan con viento de sur (escenario más frecuente) podrían causar el atrapamiento de personas durante su evacuación ya que únicamente existe una única carretera de salida o vía de escape al norte en todo el valle (Fig. 6B). Este problema se podría resolver fácilmente habilitando pistas alternativas que conecten los núcleos urbanos de este a oeste para permitir una evacuación segura.

Prácticamente la totalidad de la superficie forestal corresponde a comunales y es por tanto la Administración Forestal quien establece las condiciones técnicas en su aprovechamiento. En el caso de las claras de pinares productores, puede resultar oportuno requerir el tratamiento de restos (es decir, trituración de ramas y raberones) en las masas con elevada probabilidad de quema o próximas a núcleos urbanos. Esto puede solicitarse al maderista adjudicatario del aprovechamiento en el pliego de condiciones técnicas o realizarse después por cuenta propia una vez concluido el aprovechamiento forestal. Por su parte, la ejecución de quemas prescritas por parte de personal cualificado resulta una técnica adecuada para la reducción de combustibles en pinares a partir de edades de latizal alto y sin excesivas acumulaciones de restos. En los robledales con aprovechamiento de leñas para hogares las claras por lo bajo con un posterior apilado y troceado de restos supone el tratamiento más adecuado y extendido. En todo caso, es en los Planes Técnicos de Ordenación Forestal donde se deberían identificar los puntos estratégicos de gestión (PEG) así como las técnicas y prescripciones a seguir en cada tipo de masa. A tal efecto, el modelo de optimización que se presenta en este estudio supone una herramienta de gran utilidad para asistir en el diseño de los tratamientos capaz de generar la cartografía de detalle requerida por los gestores del territorio. Aunque nos hemos centrado en el tratamiento de combustibles forestales (masas con elevada carga y continuidad de combustibles), la gestión de pastos comunales con ganadería extensiva es una medida complementaria que ayuda a prevenir el crecimiento de matorral e incrementa la durabilidad de los tratamientos (Casasús et al., 2007; Ruiz-Mirazo et al., 2011) si los tratamientos se integran dentro de "las hierbas" (es decir, grandes recintos cercados de terreno comunal y habilitados con agua para el aprovechamiento ganadero) de los diferentes Concejos de Juslapeña. A pesar de que la longitud de llama en modelos de combustibles herbáceos no representa una gran limitación durante la extinción, la gestión con pastoreo puede ser de- 
terminante debido a que las velocidades de propagación en condiciones meteorológicas extremas (fuertes vientos y bajas humedades relativas) supera fácilmente la capacidad de extinción de los medios terrestres.

A pesar de que este estudio se centra en la gestión de combustibles, no se debe dejar de lado las medidas encaminadas a prevenir las igniciones antrópicas. En ambiente mediterráneo la mayoría de las igniciones son causadas por humanos y el desarrollo de programas de monitoreo y prevención de igniciones son también medidas prioritarias (Curt et al., 2016; Gonzalez-Olabarria et al., 2012). Nosotros hemos empleado un modelo de ocurrencia de incendios a partir del cual se pueden identificar fácilmente los lugares donde la probabilidad de ignición es especialmente elevada. Además, la identificación de las causas más frecuentes permite desarrollar protocolos con medidas preventivas específicas y que puedan ser aplicadas en los lugares que presentan una elevada probabilidad de ignición. Por ejemplo, las igniciones causadas por la maquinaria agrícola son uno de los casos más típicos en verano (cosechadoras y empacadoras). La aplicación de medidas preventivas así como la limpieza de restos vegetales en conductos y motores o la disponibilidad de equipos extintores o cubas que permitan una rápida respuesta en caso de ignición, pueden resultar decisivas para la extinción del incendio en un primer ataque (González, 2013).

Los paisajes culturales mediterráneos presentan a menudo un carácter multifuncional y el modelo de optimización empleado en este estudio permite la integración de varios objetivos y el establecimiento de prioridades o pesos en base a las necesidades determinadas en procesos de planificación (Ager et al., 2016b; Alcasena et al., 2018; Vogler et al., 2015). En última instancia, la frontera de posibilidades de producción no es más que la curva (proyección tridimensional en nuestro caso; Fig. 8) obtenida a partir de las soluciones óptimas de todas las combinaciones de pesos posibles entre objetivos. A modo ilustrativo en este estudio presentamos el mosaico de tratamientos correspondiente a los escenarios que buscan maximizar un único objetivo, así como la solución intermedia que considera un mismo peso (misma prioridad) para los tres objetivos (Fig. 9). Las mayores sinergias se observan en aquellos rodales con una mayor correspondencia espacial. El empleo de métodos de evaluación multi-criterio podría facilitar en estudios futuros la determinación de los pesos a asignar en cada objetivo y obtener así la solución de consenso más conveniente para todos los agentes territoriales implicados en la gestión, uso y disfrute del territorio (Grošelj et al., 2016; Uhde et al., 2015).

\section{Conclusiones}

En el presente estudio se demuestra cómo se pude estimar económicamente el riesgo de incendios. Además, se aplica un procedimiento de optimización espacial que permite priorizar el tratamiento estratégico de combustibles a escala de paisaje. La metodología empleada permitiría evaluar cuantitativamente los efectos para cualquier tipo de configuración espacial de tratamientos. El área de planificación (cuenca de exposición) entorno a cualquier bien de elevado valor (por ej., comunidades localizadas en la interfaz urbano-forestal) viene determinada por el potencial de gran incendio forestal. El estudio de la correspondencia espacial de soluciones para objetivos diferentes permite determinar 
las oportunidades existentes en la gestión multifuncional de los paisajes Mediterráneos. Los resultados se trasladan a una cartografía de detalle que facilita su integración en los planes de gestión forestal.

\section{Agradecimientos}

Quisiéramos agradecer al Guarderío Forestal de la Cuenca de Pamplona (DRMAyAL) y a Bomberos de Navarra su colaboración en la realización de este estudio. Los datos correspondientes a los incendios históricos fueron proporcionados por el Centro de Coordinación de la Información Nacional sobre Incendios Forestales (CCINIF), órgano del Ministerio de Agricultura y Pesca, Alimentación y Medio Ambiente responsable de coordinar la Estadística General de Incendios Forestales (EGIF). El Servicio de Riqueza Territorial y Tributos Patrimoniales, el Negociado de Suelos y Climatología y la Dirección General de Obras Públicas del Gobierno de Navarra facilitaron datos imprescindibles para la realización de este estudio. Este estudio ha sido financiado por el contrato de formación a investigadores de la Universitat de Lleida concedido a Fermín Alcasena.

\section{Referencias}

Ager, A.A., Day, M.A., Short, K.C., Evers, C.R. 2016a. Assessing the impacts of federal forest planning on wildfire risk mitigation in the Pacific Northwest, USA. Landscape and Urban Planning 147, 1-17. https://doi.org/10.1016/j.landurbplan.2015.11.007.

Ager, A.A., Day, M.A., Vogler, K. 2016b. Production possibility frontiers and socioecological tradeoffs for restoration of fire adapted forests. Journal of Environmental Management 176, 157-168. https://doi.org/10.1016/j.jenvman.2016.01.033.

Ager, A.A., Finney, M.A., McMahan, A.J., Cathcart, J. 2010. Measuring the effect of fuel treatments on forest carbon using landscape risk analysis. Natural Hazards and Earth System Sciences 10, 2515-2526. https://doi.org/10.5194/nhess-10-2515-2010.

Ager, A.A., Vaillant, N.M., Finney, M.A. 2011. Integrating fire behavior models and geospatial analysis for wildland fire risk assessment and fuel management planning. Journal of Combustion 572452, 19. http://doi.org/10.1155/2011/572452.

Ager, A.A., Vaillant, N.M., McMahan, A. 2013. Restoration of fire in managed forests: a model to prioritize landscapes and analyze tradeoffs. Ecosphere 4 (2), 29. http://doi.org/10.1890/ ES13-00007.1.

Alcasena,F.J.,Ager, A.A.,Bailey,J.D.,Pineda,N., Vega-Garcia,C.2019. Towards a comprehensive wildfire management strategy for Mediterranean areas: Framework development and implementation in Catalonia, Spain. Journal of Environmental Management 231, 303-320. https://doi.org/10.1016/j.jenvman.2018.10.027.

Alcasena, F.J., Ager, A.A., Salis, M., Day, M.A., Vega-Garcia, C. 2018. Optimizing prescribed fire allocation for managing fire risk in central Catalonia. Science of the Total Environment 4 , 872-885. https://doi.org/10.1016/j.scitotenv.2017.11.297.

Alcasena, F.J., Salis, M., Ager, A.A., Arca, B., Molina, D., Spano, D. 2015. Assessing landscape scale wildfire exposure for highly valued resources in a Mediterranean area. Environmental Management 55, 1200-1216. https://doi.org/10.1007/s00267-015-0448-6.

Alcasena, F.J., Salis, M., Ager, A.A., Castell, R., Vega-Garcia, C. 2017. Assessing wildland fire risk transmission to communities in northern Spain. Forests 8, 27. https://doi.org/10.3390/ f8020030. 
Alcasena, F.J., Salis, M., Nauslar, N.J., Aguinaga, A.E., Vega-García, C. 2016a. Quantifying economic losses from wildfires in black pine afforestations of northern Spain. Forest Policy and Economics 73, 153-167. https://doi.org/10.1016/j.forpol.2016.09.005.

Alcasena, F.J., Salis, M., Vega-García, C. 2016b. A fire modeling approach to assess wildfire exposure of valued resources in central Navarra, Spain. European Journal of Forest Research 2016b; 135, 87-107. https://doi.org/10.1007/s10342-015-0919-6.

Arca, B., Duce, P., Laconi, M., Pellizzaro, G., Salis, M., Spano, D. 2007. Evaluation of FARSITE simulator in Mediterranean maquis. International Journal of Wildland Fire 16, 563-572. https://doi.org/10.1071/WF06070.

Arca, B., Ghisu, T., Trunfio, G.A. 2015. GPU-accelerated multi-objective optimization of fuel treatments for mitigating wildfire hazard. Journal of Computational Science 11, 258-268. https://doi.org/10.1016/j.jocs.2015.08.009.

Bovio, G., Marchetti, M., Tonarelli, L., Salis, M., Vacchiano, G., Lovreglio, R., Elia, M., Fiorucci, P., Ascoli, D. 2017. Forest fires are changing: let's change the fire management strategy. Forest@ 14, 202-205.https:// doi.org/10.3832/efor2537-014.

Bradshaw, L., McCormick, E. 2000. Fire Family Plus user's guide, Version 2.0. USDA Forest Service, Ogden, UT.

Butler, B.W. 2014. Wildland firefighter safety zones: a review of past science and summary of future needs. International Journal of Wildland Fire 23, 295. http://doi.org/10.1071/ WF13021.

Calkin, D., Ager, A.A., Thompson, M., Finney, M., Lee, D.C., Quigley, T.M., McHugh, Ch.W, Riley, K.L. 2011. A comparative risk assessment framework for wildland fire management: the 2010 cohesive strategy science report. USDA Forest Service, Rocky Mountain Research Station, Fort Collins, CO, 63 pp.

Calkin, D.E., Cohen, J.D., Finney, M.A., Thompson, M.P. 2014. How risk management can prevent future wildfire disasters in the wildland-urban interface. Proceedings of the National Academy of Sciences 111, 746-751 https://doi.org/10.1073/pnas.1315088111.

Casasús, I., Bernués, A., Sanz, A., Villalba, D., Riedel, J.L., Revilla, R. 2007. Vegetation dynamics in Mediterranean forest pastures as affected by beef cattle grazing. Agriculture, Ecosystems \& Environment 121,365-370. https://doi.org/10.1016/j.agee.2006.11.012.

Castellnou, M., Miralles, M. 2009. The changing face of wildfires. Crisis Response 5, 56-57.

Cervera, T., Pino, J., Marull, J., Padró, R., Tello, E. 2019. Understanding the long-term dynamics of forest transition: From deforestation to afforestation in a Mediterranean landscape (Catalonia, 1868-2005). Land Use Policy 80, 318-331. https://doi.org/10.1016/j. landusepol.2016.10.006.

Cohen, J. 2008. The wildland-urban interface fire problem: a consequence of the fire exclusion paradigm. Forest History Today. Fall, 20-26.

Costa, P., Castellnou, M., Larrañaga, A., Miralles, M., Daniel, K. 2011. Prevention of large wildfires using the fire types concept. Cerdanyola del Vallès, Barcelona, Spain.

Costafreda-Aumedes, S, Comas, C., Vega-García, C. 2016. Spatio-Temporal Configurations of Human-Caused Fires in Spain through Point Patterns. Forests 7 (9), 185.

Costafreda-Aumedes, S., Vega-García, C., Comas, C. 2018. Improving fire season definition by optimized temporal modelling of daily human-caused ignitions. Journal of Environmental Management 217, 90-99. https://doi.org/10.1016/j.jenvman.2018.03.080.

Curt. T., Fréjaville, T., Lahaye, S. 2016. Modelling the spatial patterns of ignition causes and fire regime features in southern France: implications for fire prevention policy. International Journal of Wildland Fire 25, 785-796. https://doi.org/10.1071/WF15205.

Dalkey, N.C., Helmer, O. 1963. An experimental application of the Delphi method to the use of experts. Management Science 9, 458-468. 
Duane, A., Brotons, L. 2018. Synoptic weather conditions and changing fire regimes in a Mediterranean environment. Agricultural and Forest Meteorology 253-254, 190-202. https:// doi.org/10.1016/j.agrformet.2018.02.014.

Fahlman, S.E., Lebiere, C. 1990. The cascade-correlation learning architecture. In: D.S. Touretzky (Ed.), Advances in Neural Information Processing Systems. Morgan Kaufmann, San Francisco, USA, pp. 524-532.

Fernandes, P.M. 2009. Combining forest structure data and fuel modelling to classify fire hazard in Portugal. Annals of Forest Science 66, 415. https://doi.org/10.1051/forest/2009013.

Fernandes P.M. 2013. Fire-smart management of forest landscapes in the Mediterranean basin under global change. Landscape and Urban Planning 110, 175-182. https://doi.org/10.1016/j. landurbplan.2012.10.014.

Fernandes, P.M., Fernandes, M.M., Loureiro, C. 2012. Survival to prescribed fire of plantationgrown Corsican black pine in northern Portugal. Annals of Forest Science 69, 813-820. https://doi.org/10.1007/s13595-012-0211-6.

Fernandes, P.M., Vega, J.A., Jiménez, E., Rigolot, E. 2008. Fire resistance of European pines. Forest Ecology and Management 256: 246-255.https://doi.org/10.1016/j.foreco.2008.04.032.

Finney, M.A. 2002. Fire growth using minimum travel time methods. Canadian Journal of Forest Research 32, 1420-1424. https://doi.org/10.1139/x02-068.

Finney, M.A. 2004. Chapter 9, landscape fire simulation and fuel treatment optimization. In: J.L. Hayes, A.A. Ager, J.R. Barbour (Eds), Methods for integrated modeling of landscape change: Interior Northwest Landscape Analysis, System. US Forest Service Gen. Tech. Rep., PNW-GTR-610. Portland, Oregon, pp. 117-131.

Finney, M.A. 2005. The challenge of quantitative risk analysis for wildland fire. Forest Ecology and Management 211,97-108. https://doi.org/10.1016/j.foreco.2005.02.010.

Finney, M.A. 2006. An overview of FlamMap fire modeling capabilities. In: P.L. Andrews, B.W. Butler (Eds), Fuels Management-How to Measure Success. Proceedings RMRS-P-41. USDA Forest Service, Rocky Mountain Research Station, Fort Collins, CO, pp. 213-220.

Finney, M.A. 2007. A computational method for optimizing fuel treatment location. International Journal of Wildland Fire 16, 702-711.

Finney, M.A., Seli, R.C., McHugh, C.W., Ager, A.A., Bahro, B., Agee, J.K. 2006. Simulation of long-term landscape-level fuel treatment effects on large wildfires. In: P.L. Andrews, B.W. Butler (Eds.), Fuels Management-How to Measure Success. Proceedings RMRS-P-41. USDA Forest Service, Rocky Mountain Research Station, Fort Collins, CO, pp. 125-147.

Finney, M.A., Seli, R.C., McHugh, C.W., Ager, A.A., Bahro, B., Agee, J.K. 2007. Simulation of long-term landscape-level fuel treatment effects on large wildfires. International Journal of Wildland Fire 16, 712-727.

González-Olabarria, J.R., Brotons, L., Gritten, D., Tudela, A., Teres, J.A. 2012. Identifying location and causality of fire ignition hotspots in a Mediterranean region. International Journal of Wildland Fire 21, 905-914. https://doi.org/10.1071/WF11039.

González-Olabarria, J.R., Reynolds, K.M., Larrañaga, A., García-Gonzalo, J., Busquets, E., Pique, M. 2019. Strategic and tactical planning to improve suppression efforts against large forest fires in the Catalonia region of Spain. Forest Ecology and Management 432, 612-622. https:// doi.org/10.1016/j.foreco.2018.09.039.

González-Olabarria, J.R., Rodríguez, F., Fernández-Landa, A., Mola-Yudego, B. 2012. Mapping fire risk in the Model Forest of Urbión (Spain) based on airborne LiDAR measurements. Forest Ecology and Management 282, 149-156. https://doi.org/10.1016/j. foreco.2012.06.056.

González, L.A. 2013. La maquinaria agrícola y los incendios forestales. Alerta! 2, 29-34. 
Grošelj, P., Hodges, D.G., Stirn, L.Z. 2016. Participatory and multi-criteria analysis for forest (ecosystem) management: A case study of Pohorje, Slovenia. Forest Policy and Economics 71, 80-86. https://doi.org/10.1016/j.forpol.2015.05.006.

Jahdi, R., Salis, M., Darvishsefat, A.A., Alcasena, F., Mostafavi, M.A., Etemad, V., Lozano, O., Spano, D. 2016. Evaluating fire modelling systems in recent wildfires of the Golestan National Park, Iran. Forestry 89, 136-149. https://doi.org/10.1093/forestry/cpv045.

Lasanta, T., Nadal-Romero, E., García-Ruiz, J.M. 2019. Clearing shrubland as a strategy to encourage extensive livestock farming in the Mediterranean mountains. Cuadernos de Investigación Geográfica 45. http://doi.org/10.18172/cig.3616.

Lovreglio, R., Leone, V., Giaquinto, P., Notarnicola, A. 2010. Wildfire cause analysis: four case-studies in southern Italy. iForest - Biogeosciences and Forestry 3, 8-15. http://doi. org/10.3832/ifor0521-003.

Lozano, O., Salis, M., Ager, A.A., Arca, B., Alcasena, F.J., Monteiro, A., 2017. Assessing climate change impacts on wildfire exposure in Mediterrean areas. Risk Analysis 37, 1898-1916. https://doi.org/10.1111/risa.12739.

MAAуMA. 2015. Estadística General de Incendios Forestales. Centro de Coordinación de la Información Nacional sobre Incendios Forestales. Ministerio de Agricultura, Alimentación y Medio Ambiente, Madrid.

Madrigal, J., Fernández-Migueláñez, I., Hernando, C., Guijarro, M., Vega-Nieva, D.J., Tolosana, E. 2016. Does forest biomass harvesting for energy reduce fire hazard in the Mediterranean basin? a case study in the Caroig Massif (Eastern Spain). European Journal of Forest Research 136, 13-26. https://doi.org/10.1007/s10342-016-1004-5.

Marino, E., Ranz, P., Tomé, J.L., Noriega, M.Á., Esteban, J., Madrigal, J. 2016. Generation of high-resolution fuel model maps from discrete airborne laser scanner and Landsat-8 OLI: A low-cost and highly updated methodology for large areas. Remote Sensing of Environment 187, 267-280. https://doi.org/10.1016/j.rse.2016.10.020.

Martínez, J., Vega-García, C., Chuvieco, E. 2009. Human-caused wildfire risk rating for prevention planning in Spain. Journal of Environmental Management 90, 1241-1252. https:// doi.org/10.1016/j.jenvman.2008.07.005.

McGaughey, R.J. 2018. FUSION/LDV: Software for LIDAR Data Analysis and Visualization. USDA Forest Service, Pacific Northwest Research Station, Seattle, 207 pp.

Meddour-Sahar, O., Meddour, R., Leone, V., Lovreglio, R., Derridj, A. 2013. Analysis of forest fires causes and their motivations in northern Algeria: the Delphi method. iForest Biogeosciences and Forestry 6, 247-254. https://doi.org/10.3832/ifor0098-006.

Miller, C., Ager, A.A. 2013. A review of recent advances in risk analysis for wildfire management. International Journal of Wildland Fire 22, 1-14. https://doi.org/10.1071/WF11114.

Moya, D., Alfaro-Sánchez, R., López-Serrano, F., Dadi, T., Hernández-Tecles, E., Ferrandis, P., de las Heras, J. 2014. Post-fire management of mediterranean forests: Carbon storage in regenerated areas in eastern iberian peninsula. Cuadernos de Investigación Geográfica 40, 371-386. http://doi.org/10.18172/cig.2565.

Nelson, R.M. 2000. Prediction of diurnal change in 10-h fuel stick moisture content. Canadian Journal of Forest Research 30, 1071-1087. https://doi.org/10.1139/x00-032.

Oliveira, T.M., Barros, A.M.G., Ager, A.A., Fernandes, P.M. 2016. Assessing the effect of a fuel break network to reduce burnt area and wildfire risk transmission. International Journal of Wildland Fire 25, 619-632. https://doi.org/10.1071/WF15146.

Palaiologou, P., Ager, A.A., Nielsen-Pincus, M., Evers, C., Kalabokidis, K. 2018. Using transboundary wildfire exposure assessments to improve fire management programs: a case study in Greece. International Journal of Wildland Fire, 27, 501-513. https://doi.org/10.1071/ WF17119. 
Pausas, J.G., Fernández-Muñoz, S. 2012. Fire regime changes in the Western Mediterranean Basin: from fuel-limited to drought-driven fire regime. Climatic Change 110, 215-226. https://doi. org/10.1007/s10584-011-0060-6.

Paveglio, T.B., Prato, T., Edgeley, C., Nalle, D. 2016. Evaluating the characteristics of social vulnerability to wildfire: demographics, perceptions, and parcel characteristics. Environmental Management 58, 534-548. https://doi.org/10.1007/s00267-016-0719-х.

Penman, T.D., Nicholson, A.E., Bradstock, R.A., Collins, L., Penman, S.H., Price, O.F. 2015. Reducing the risk of house loss due to wildfires. Environmental Modelling \& Software 67, 12-25. https://doi.org/10.1016/j.envsoft.2014.12.020.

Peterson, D.L., Ryan, K.C. 1986. Modeling postfire conifer mortality for long-range planning. Environmental Management 10, 797-808.

Poyatos, R., Latron, J., Llorens, P. 2003. Land Use and Land Cover Change After Agricultural Abandonment: The Case of a Mediterranean Mountain Area (Catalan Pre-Pyrenees). Mountain Research and Development 23, 362-369. https://doi.org/10.1659/02764741(2003)023[0362:LUALCC]2.0.CO;2.

Prats, S., Malvar, M., Martins, M.A.S., Keizer, J.J. 2014. Post-fire soil erosion mitigation: a review of the last research and techniques developed in Portugal. Cuadernos de Investigación Geográfica 40, 403-427. http://doi.org/10.18172/cig.2519.

Rodrigues, M., De la Riva, J. 2014. An insight into machine-learning algorithms to model humancaused wildfire occurrence. Environmental Modeling and Assessment 57, 192-201. https:// doi.org/10.1016/j.envsoft.2014.03.003.

Ruiz-Mirazo, J., Robles, A.B., González-Rebollar, J.L. 2011. Two-year evaluation of fuelbreaks grazed by livestock in the wildfire prevention program in Andalusia (Spain). Agriculture, Ecosystems \& Environment 141, 13-22. https://doi.org/10.1016/j.agee.2011.02.002.

Salis, M., Ager, A.A., Arca, B., Finney, M.A., Bacciu, V., Duce, P., Spano, D. 2012. Assessing exposure of human and ecological values to wildfire in Sardinia, Italy. International Journal of Wildland Fire 22, 549-565. https://doi.org/10.1071/WF11060.

Salis, M., Arca, B., Alcasena, F., Arianoutsou, M., Bacciu, V., Duce. P., Duguy, B., Koutsias, N., Mallinis, G., Mitsopoulos, I. Moreno, J.M., Pérez, J.R., Urbieta, I., Xystrakis, F., Zavala, G., Spano, D. 2016a. Predicting wildfire spread and behaviour in Mediterranean landscapes. International Journal of Wildland Fire 25, 1015-1032. https://doi.org/10.1071/WF15081.

Salis, M., Laconi, M., Ager, A.A., Alcasena, F., Arca, B., Lozano, O., Fernandez, A., Spano, D. 2016b. Evaluating alternative fuel treatment strategies to reduce wildfire losses in a Mediterranean area. Forest Ecology and Management 368, 207-221. https://doi.org/10.1016/j. foreco.2016.03.009.

Salis, M., Del Guiudice, L., Arca, B., Ager, A.A., Alcasena, F., Lozano, O., Bacciu, V., Spano, D., Ducea, P. 2018. Modeling the effects of different fuel treatment mosaics on wildfire spread and behavior in a Mediterranean agro-pastoral area. Journal of Environmental Management 212, 490-505. https://doi.org/10.1016/j.jenvman.2018.02.020.

San-Miguel-Ayanz, J., Durrant, T., Boca, R., Libertà, G., Branco, A., De Rigo, D., Ferrari, D., Maianti, P., Vivancos, T., Schulte, E., Loffler, P. 2017. Forest Fires in Europe, Middle East and North Africa 2016. Joint Research Centre, European Comission, Luxenburg, 2017, $120 \mathrm{pp}$.

San-Miguel-Ayanz, J., Moreno, J.M., Camia, A. 2013. Analysis of large fires in European Mediterranean landscapes: Lessons learned and perspectives. Forest Ecology and Management 294, 11-22. https://doi.org/10.1016/j.foreco.2012.10.050.

Scott, J.H., Burgan, R.E. 2005. Standard fire behavior fuel models: a comprehensive set for use with Rothermel's surface fire spread model. USDA Forest Service, Rocky Mountain Research Station, Fort Collins, CO, 72 pp. 
Scott, J.H., Thompson, M.P., Calkin, D.E. 2013. A wildfire risk assessment framework for land and resource management. USDA Forest Service, Rocky Mountain Research Station, 83 pp.

Scott, J.H., Thompson, M.P., Gilbertson-Day, J.W. 2016. Examining alternative fuel management strategies and the relative contribution of National Forest System land to wildfire risk to adjacent homes - A pilot assessment on the Sierra National Forest, California, USA. Forest Ecology and Management 362, 29-37. https://doi.org/10.1016/j.foreco.2015.11.038.

Seijo, F., Gray, R. 2012. Pre-Industrial Anthropogenic Fire Regimes in Transition: The Case of Spain and its Implications for Fire Governance in Mediterranean Type Biomes. Human Ecology Review 19, 58-69.

Syphard, A.D., Brennan, T.J., Keeley, J.E. 2014. The role of defensible space for residential structure protection during wildfires. International Journal of Wildland Fire 23, 1165. https:// doi.org/10.1071/WF13158.

Thompson, M., Calkin, D., Gilbertson-Day, J., Ager, A.A. 2011. Advancing effects analysis for integrated, large-scale wildfire risk assessment. Environmental Monitoring and Assessment 179, 217-239. https://doi.org/10.1007/s10661-010-1731-x.

Thompson, M.P., Scott, J., Kaiden, J.D., Gilbertson-Day, J.W. 2013. A polygon-based modeling approach to assess exposure of resources and assets to wildfire. Natural Hazards 67, 627644. https://doi.org/10.1007/s11069-013-0593-2.

Uhde, B., Hahn, W.A., Griess, V.C., Knoke, T. 2015. Hybrid MCDA Methods to Integrate Multiple Ecosystem Services in Forest Management Planning: A Critical Review. Environmental Management 56, 373-88. https://doi.org/10.1007/s00267-015-0503-3.

Vega-García, C., Woodard, P.M., Titus, S.J., Adamowicz, W.L., Lee, B.S. 1995. A logit model for predicting the daily occurrence of human caused forest-fires. International Journal of Wildland Fire 5, 101-111.

Vogler, K.C., Ager, A.A., Day, M.A., Jennings, M., Bailey, J.D. 2015. Prioritization of forest restoration projects: tradeoffs between wildfire protection, ecological restoration and economic objectives. Forests 6, 4403-4420. https://doi.org/10.3390/f6124375. 